\title{
Effects of Nutritional Interventions during Pregnancy on Infant and Child Cognitive Outcomes: A Systematic Review and Meta-Analysis
}

\author{
Rachael M. Taylor 1,2,3,*, Shanna M. Fealy ${ }^{2,4}$, Alessandra Bisquera ${ }^{3,5}$, Roger Smith ${ }^{1,2,3}$, \\ Clare E. Collins ${ }^{3,6,7}$, Tiffany-Jane Evans ${ }^{3,5}$ and Alexis J. Hure $2,3,8$ \\ 1 Priority Research Centre for Reproductive Science, University of Newcastle, Callaghan, \\ NSW 2308, Australia; roger.smith@newcastle.edu.au \\ 2 Faculty of Health and Medicine, School of Medicine and Public Health, University of Newcastle, Callaghan, \\ NSW 2308, Australia; shanna.fealy@newcastle.edu.au (S.M.F.); alexis.hure@newcastle.edu.au (A.J.H.) \\ 3 Hunter Medical Research Institute, 1 Kookaburra Circuit, New Lambton Heights, NSW 2305, Australia; \\ alessandra.bisquera@kcl.ac.uk (A.B.); clare.collins@newcastle.edu.au (C.E.C.); \\ tiffany.evans@hmri.org.au (T.-J.E.) \\ 4 Faculty of Health and Medicine, School of Nursing \& Midwifery, University of Newcastle, Callaghan, \\ NSW 2308, Australia \\ 5 Clinical Research Design IT and Statistical Support (CReDITSS) Unit, Hunter Medical Research Institute, \\ 1 Kookaburra Circuit, New Lambton Heights, NSW 2305, Australia \\ 6 Faculty of Health and Medicine, School of Health Sciences, University of Newcastle, Callaghan, \\ NSW 2308, Australia \\ 7 Priority Research Centre in Physical Activity and Nutrition, University of Newcastle, Callaghan, \\ NSW 2308, Australia \\ 8 Priority Research Centre for Gender, Health and Ageing, University of Newcastle, Callaghan, \\ NSW 2308, Australia \\ * Correspondence: c3093931@uon.edu.au or rachael.m.taylor@uon.edu.au; Tel.: +61-2406-2784
}

Received: 19 October 2017; Accepted: 13 November 2017; Published: 20 November 2017

\begin{abstract}
Background: Epidemiological studies have demonstrated that folate, iodine and iron intake during pregnancy impacts on foetal brain development and cognitive function. However, in human studies, the relationship with other dietary nutrients is less clear. Objective: This systematic review aims to critically appraise the current literature and meta-analyses results from nutritional interventions during pregnancy that aimed to optimise infant and child cognitive outcomes. Design: Ten electronic databases were searched for articles published up to August 2017. The search was limited to articles published in English. Randomised controlled trials (RCTs) testing the impact of any nutritional intervention (dietary counselling, education, nutrient supplementation, fortified foods and/or foods) during pregnancy on cognitive outcomes of children ( $<10$ years old). Two independent reviewers assessed study eligibility and quality using the American Dietetic Association quality criteria checklist for primary research. Standardised mean differences were used for nine cognitive domains to measure effects for meta-analyses. Results: A total of 34 RCTs were included (21 studies included children aged less than 35 months, 10 studies included children aged 36-60 months and 3 studies included children aged 61-119 months). The types of nutritional interventions included nutrient supplements, whole foods, fortified foods and nutrition education. The following nine cognition outcomes: attention, behaviour, crystallised intelligence, fluid intelligence, global cognition, memory, motor skills, visual processing, and problem solving were not significantly impacted by nutritional interventions, although $65 \%$ of studies conducted post-hoc data analyses and were likely to be underpowered. Although, long chain polyunsaturated fatty acids (LCPUFA) supplementation was associated with a marginal increase in crystallised intelligence (Effect size (ES): $0.25 ; 95 \%$ confidence interval $(95 \% \mathrm{CI}):-0.04,0.53)$, the effect was not statistically significant $(p=0.09)$, with significant study heterogeneity $(p=0.00)$. Conclusions: LCPUFA supplementation may be associated with an
\end{abstract}


improvement in child crystallised intelligence, however further research is warranted. The remaining eight cognition domains were not significantly impacted by maternal nutritional interventions.

Keywords: behaviour; child; cognition; cognitive function; infant; nutrition; pregnancy; supplement

\section{Introduction}

Adequate nutrition during the prenatal period and early years of life is essential for brain development and cognitive function. Approximately 28 days after conception the neural plate folds and fuses, forming the neural tube, which gives rise to the development of the foetal brain [1]. Adequate folate from the maternal diet during this period is essential for the formation of the neural tube, a deficiency in this nutrient can adversely affect brain development, resulting in neural tube defects, spina bifida and encephalocoele [2-6]. Following the formation of the neural tube, neurodevelopmental processes including, cell proliferation and migration occur during gestation, while neurogenesis, synaptic formation and myelination continue until late adolescence [7]. Iodine is necessary for neural cell migration and differentiation, synaptogenesis and myelination [8], while dietary iron is necessary for neurogenesis and dopamine production [9-11]. Deficiency in these nutrients are known to compromise brain development and cause significant cognitive impairment in the offspring [8,12]. The importance of an adequate intake of folate, iodine and iron during pregnancy for foetal brain development has been well explored; however, the developmental role of other dietary nutrients (e.g., zinc, long-chain polyunsaturated fatty acids (LCPUFAs)) remains unclear from human studies.

The link between early-life nutrition and child cognitive function is difficult to establish because the brain is a heterogeneous organ consisting of multiple anatomic regions (e.g., the hippocampus, striatum, cortex) and neurodevelopmental processes (e.g., synaptogenesis, myelination) with distinct developmental trajectories that span and peak at different times $[13,14]$. For example, myelination commences at 12-14th week of gestation and occurs at a peak rate during the first two years of life, but continues until adulthood [15]. The prefrontal cortex, which has a prominent role in higher cognitive control, develops in growth spurts during the first two years of life, once again between seven and nine years of age and also at approximately 15 years of age [16,17]. The effect of nutrition on cognitive function depends on the nutrients involved and the maturation stage of the brain. These nutrient effects on brain structure and function may occur in the short-term, while others may not be apparent until full maturation is reached [18].

Optimising brain development and cognitive function holds major long-term consequences for individuals and societies. Cognitive function affects academic performance and the level of education attained [19]. Higher educational attainment is related to a lower burden of disease, due to greater health care access and healthy lifestyle behaviours [20]. Suboptimal cognition during childhood has been associated with an increased risk of adolescent delinquency [21-23] and adult violent criminality [21,24-26]. Incarceration can cause permanent cognitive damage, which holds major implications for future education, employment and health [27]. Strategies that aim to maximise the cognitive performance of children are therefore important for public health policy and nutrition during pregnancy.

This systematic review and meta-analysis aimed to determine whether nutritional intervention/s during pregnancy alter cognitive outcomes during infancy and late childhood. Larson et al. [28] analysed the impact of maternal nutritional interventions on child cognition, however this review focused on short-term cognitive outcomes in children under the age of two years in developing countries. Therefore, a systematic review that considers the impact of maternal nutritional interventions on long-term cognitive outcomes of children living in developed and developing countries is warranted to provide the most comprehensive evaluation of the literature in this area. 


\section{Methods}

The review protocol was developed using The Cochrane Handbook for Systematic Reviews of Interventions. The Preferred Reporting Items for Systematic Review Meta-Analyses (PRISMA) [29] was applied. Similar methods have been followed previously by Gresham et al. [30]. The review protocol can be found in Supplementary Figure S1.

\subsection{Search Strategy}

A search strategy was devised with the assistance of a medical research librarian (DB). The final electronic literature search was conducted on August 2017, without date limits, using Medline, Pre-Medline, Embase, PsycInfo and Maternity and Infant Care via Ovid (http://www.ovid. com/), Scopus (http:/ / www.scopus.com/), Proquest (http:/ /www.proquest.com/), Web of Science (http:/ / apps.webofknowledge.com) and Cumulative Index to Nursing and Allied Health Literature via EBSCO (http:/ / www.ebsco.com/cinahl). The Cochrane Library was also searched separately to identify any similar systematic reviews that have been conducted previously. The following keywords were used: pregnan ${ }^{*}$, cognit $^{*}$, neurodevelopment, infant ${ }^{*}$, child $^{*}$, randomised control ${ }^{*}$ trial, clinical trial, food $^{*}$ nutrition and supplement* Full details of the search strategy tailored for each database can be found in Supplementary Table S1. The Boolean operation (i.e., AND, OR) was used to combine keywords that were searched as free text in the title, abstract or topics from all papers. All searches were limited to human studies published in English and citations were downloaded into the reference manager program ENDNOTE X6.v (New York, NY, USA: Thomson Reuters 2012). The keywords: mental development and motor development, were searched in all databases separately, to ensure that no eligible studies were missed from the initial search strategy.

\subsection{Study Selection}

Eligibility of the retrieved publications was assessed by two independent reviewers (RMT and SMF) as recommended by PRISMA [29]. Publication title and abstracts were evaluated against the inclusion and exclusion criteria (Table 1) Publications were excluded by a hierarchical approach based on the study design, population, intervention and outcomes. Publications meeting the initial eligibility screening were retrieved in full and were then subjected to a second round of screening by the same two reviewers to determine the final inclusion or exclusion. Assessment discrepancies between reviewers were resolved by discussion or assessment by a third party independent reviewer (AJH). The reference lists of the included publications were searched separately to identify any relevant articles that were not detected by the electronic search strategy. The abstract and then the full texts were retrieved for consideration. After the retrieval of the full text a final decision was made about the eligibility of the publication by the first reviewer (RMT).

Table 1. Inclusion and exclusion criteria for selecting studies.

\begin{tabular}{ccccc}
\hline Criteria & Study Design & Population & Intervention & Outcome \\
\hline Include & $\begin{array}{c}\text { Randomised or } \\
\text { pseudorandomised } \\
\text { controlled trials } \\
\text { of any date }\end{array}$ & $\begin{array}{c}\text { Pregnant women of } \\
\text { any age or ethnicity }\end{array}$ & $\begin{array}{c}\text { Dietary intervention/s, including } \\
\text { dietary counselling and education } \\
\text { as well as food/s, fortified foods } \\
\text { or nutrient supplement/s }\end{array}$ & $\begin{array}{c}\text { Measures cognitive } \\
\text { outcomes of infants and } \\
\text { children using cognitive } \\
\text { assessment tests }\end{array}$ \\
\cline { 2 - 6 } & & Singleton pregnancies & $\begin{array}{c}\text { Dietary intervention/s provided } \\
\text { during pregnancy (exclusively) or } \\
\text { intervention/s commencing } \\
\text { during gestation and continued } \\
\text { during lactation }\end{array}$ & $\begin{array}{c}\text { Cognition is measured } \\
\text { after birth in children } \\
<10 \text { years of age }\end{array}$ \\
\hline Exclude & $\begin{array}{c}\text { All other } \\
\text { designs including } \\
\text { animal studies }\end{array}$ & Women that are \\
\cline { 2 - 6 } & & not pregnant & $\begin{array}{c}\text { Dietary intervention/s are not } \\
\text { provided to pregnant women }\end{array}$ & $\begin{array}{c}\text { Cognitive outcomes are } \\
\text { not measured in children } \\
\text { after pregnancy }\end{array}$ \\
\hline
\end{tabular}




\subsection{Eligibility Criteria}

Table 1 outlines the inclusion and exclusion criteria for this systematic review. Studies that delivered nutritional interventions to the mother during pregnancy (exclusively) or during pregnancy plus the post-natal period ( $<10$ years) were included. This review included studies that measured cognition in children up to nine years of age because the effect of nutritional interventions may not be apparent until later life [18]. In addition, cognition assessment tests in older children ( $>7$ years) are a more reliable predictor of adult cognitive function [31]. Nutritional interventions that commenced after pregnancy were excluded. This review included studies that measured cognition (as a primary or secondary outcome) in infants and young children using one or more cognition assessment tests or subtests. Cognition domains were based on the Cattell-Horn-Carroll (CHC) Theory of Intelligence [32]. The domains fluid and crystallised intelligence, specified in the CHC model [32], were combined to form the global cognition domain for cognition assessment tests that reported an overall global composite score. Verbal composite scores were grouped in the crystallised intelligence domain and non-verbal composite scores were grouped in the fluid intelligence domain. Short-term memory and long-term retrieval domains were combined to form an overall memory domain. The remaining six cognition domains of the $\mathrm{CHC}$ model [32] (quantitative reasoning, reading and writing ability, visual processing, auditory processing, processing speed and correct/decision speed) were included. Attention, motor skills and behaviour were also analysed because these domains are commonly analysed in cognition assessment tests. For example, the Intergrowth-21st Neurodevelopment Assessment reports child cognitive scores for the domains attention, motor skills and behaviour [33]. In addition, Bayley Scales of Infant development (third edition) includes a behaviour rating scale, which measures behavioural factors including orientation/engagement and emotional regulation [34]. This review included the outcome visual processing that focuses on both the response and comprehension of visual stimuli. Visual acuity which primarily focuses on vision clarity, was not reported as an outcome in this review. Similar methods have been described previously by Eilander et al. [35].

\subsection{Quality Assessment}

Articles considered eligible were assessed for methodological quality in their reporting using the American Dietetic Association (ADA) Quality Criteria Checklist for Primary Research [36] which is applicable to evaluating the validity of nutrition studies. The quality of the articles was assessed by two independent reviewers (RMT and SMF) and assessment discrepancies between reviewers, were managed through discussion or resolved by the third independent reviewer (AJH). The Quality Criteria Checklist [36] rates the study design and execution, as well as the risk of bias using ten validity questions, including four priority questions which must be satisfactory to gain a positive rating. Based on the responses to these questions, the checklist assigns a quality rating: positive (answered "yes" to six or more validity questions, including all four priority questions), negative (answered "no" to six or more validity questions) or neutral (answered "no" to one or more of the four priority criteria questions) [36]. No studies were given a negative rating in this review, therefore no study exclusions were made.

\subsection{Data Extraction}

The first reviewer (RMT) extracted relevant data from the included studies using an Excel spreadsheet. The Organisation for Economic Co-operation and Development (OECD) criteria [37] were used to classify country income into four categories: low, lower-middle, higher-middle and high. Nutritional interventions were divided into six categories; single nutrient supplement, multiple micronutrient supplement, fortified foods, foods, supplements with foods, supplements with foods and nutrition education or no nutrition intervention (control group). For the purpose of this review a multiple micronutrient supplement was defined as two or more nutrients which is consistent with 
other authors [38-40]. The cognition outcomes data included the mean, standard deviation, confidence intervals, minimum and maximum range and odds ratio. Corresponding authors were emailed if additional data or clarification was required.

\subsection{Data Synthesis}

The nutritional interventions provided across the included studies were grouped together according to the nutrient supplied and the type of intervention (i.e., supplement, dietary changes). The cognition outcomes reported were organised into related cognition domains and the type of scoring (i.e., subtest or composite score). Thirteen cognition domain categories were derived: (i) fluid intelligence; (ii) crystallised intelligence; (iii) quantitative knowledge; (iv) memory; (v) visual processing; (vi) auditory processing; (vii) processing speed; (viii) correct decision speed; (ix) reading and writing; (x) global cognition; (xi) attention; (xii) motor skills; (xiii) behaviour.

\subsection{Statistical Analysis}

The aim of meta-analysis was to present a single effect size for the 13 specified cognition scores. The standardised mean difference (SMD) was used to measure the effect considering that all outcomes were measured using different cognition assessment tests and reporting units. The SMD, also known as Cohen's d, is defined as the difference between the mean of the intervention and control group divided by the standard deviation of the data. Methods to convert other units (e.g., Odds ratio) to SMD are described in Sanchez-Meca et al. [41]. Studies that presented multiple cognition outcomes within the same cognition domain, multiple time-points, or multiple interventions for multiple micronutrient supplementation with only a single control group, the respective method described by Borenstein et al. [42] was used to calculate a summary SMD and standard error which accounts for non-independence within each study. As the correlations between outcomes were not reported by the studies, the correlation was assumed to be 0.5 , and sensitivity analyses were performed using a higher (0.8) or lower (0.2) correlation to assess the changes in the standard error.

Supplementary Table S2 shows an example of how the composite effect size was created. A random effects meta-analytic model (using the method of DerSimonian and Laird [43]) was conducted for each cognitive outcome, where the observed effect sizes are regarded to vary due to sampling variance. A fixed effects model using inverse variance weights was also conducted for secondary analysis. The heterogeneity and total variation of studies were analysed using the $I_{2}$ statistic [44]. Funnel plots were used to assess publication bias. Subgroup analysis was performed for each outcome by the nutrient of interest. A sensitivity analysis with a random effects model was conducted in which the studies were stratified by country income. The statistical analysis was conducted using the metan command in the statistical software package STATA 13 (College Station, TX, USA: StataCorp LP 2013).

\section{Results}

\subsection{Search Results}

A total of 1195 publications (excluding duplicates, $n=439$ ) were assessed against the inclusion and exclusion criteria (Figure 1). Seven included publications were identified from hand searching the reference lists of included publications detected from the initial search strategy. These articles all used the keyword mental development, which was not included separately. This systematic review resulted in 34 included publications after inclusion and exclusion criteria were applied to full texts. 


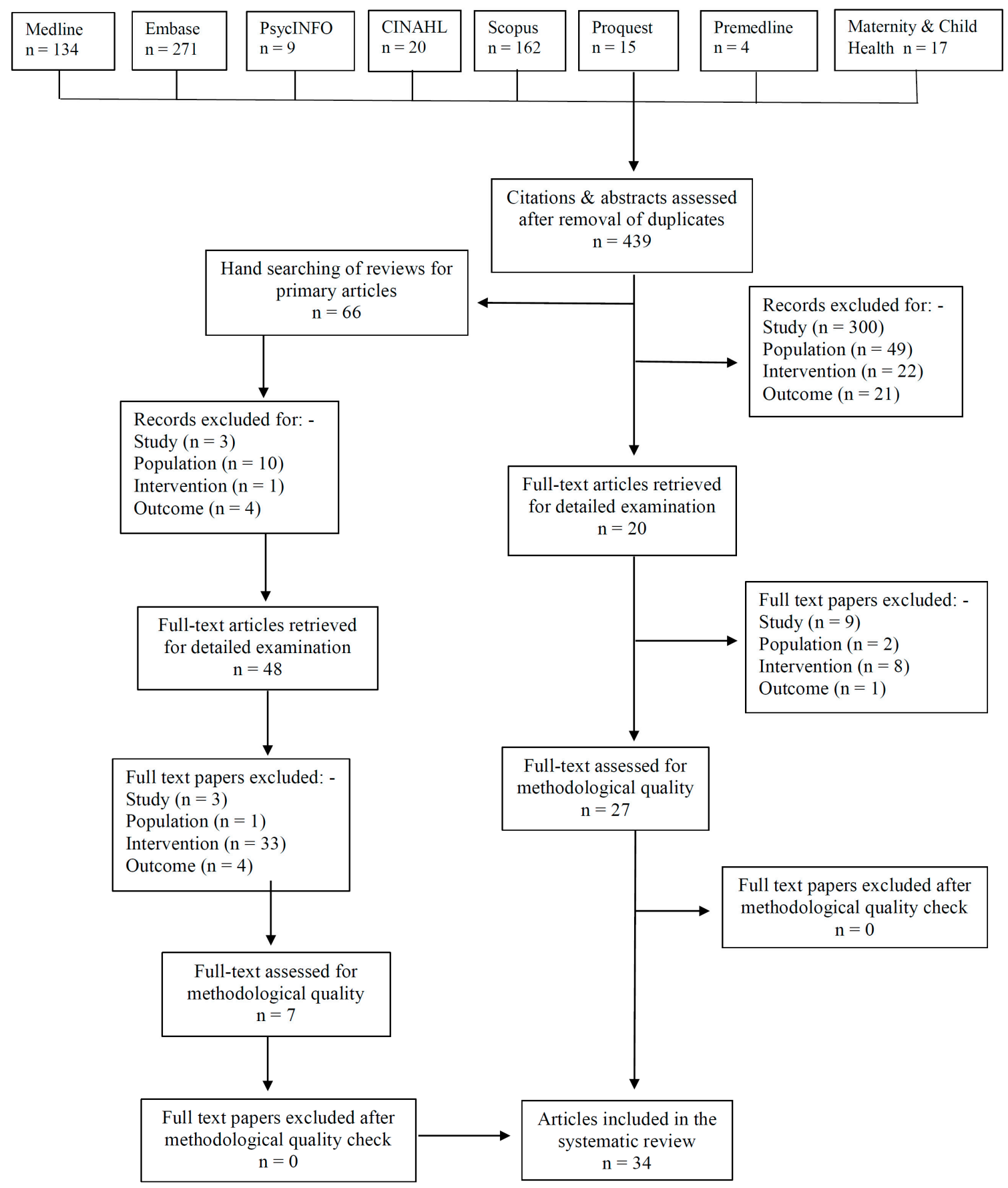

Figure 1. Flow chart for study selection process.

\subsection{Quality}

A summary of the ADA quality assessment including participant selection, handling of withdrawals and the use of study blinding for each trial is presented in Supplementary Table S3. Direct (e.g., blood, urine tests) intervention compliance measures were used in 12 out of 34 studies [45-56]. Indirect (e.g., pill count, self-reported) intervention compliance measures were used in 15 out of 34 studies [57-71]. The attrition rate ranged from $0-99 \%$ indicating the potential for bias with a loss to follow-up. Post-hoc data analysis was used in 22 studies [46,47,49-51,53,55-58,60,61,63-67,69,72-75]. 


\subsection{Description of Studies}

Table 2 summarises the characteristics of the included studies. The earliest publication was from 1979 [76] and the latest from 2017 [72,75]. A summary of the studies from the OECD is provided in Supplementary Table S5. In summary, 50\% of studies were from high-income countries, 29\% of studies were from middle-income countries and $21 \%$ of studies were from low-income countries.

\subsection{Participants}

The study populations were mostly healthy pregnant women, except one publication [61] which included pregnant women with human immunodeficiency virus type 1 (HIV-1), although pregnant women were excluded if they were diagnosed with acquired immunodeficiency syndrome (AIDS) according to the World Health Organisation (WHO) definition [77].

\subsection{Intervention}

A summary of the nutritional interventions of the studies is provided in Supplementary Table S4. In summary, $76 \%$ of studies provided a nutrient supplement exclusively for the study intervention, no studies provided nutrition counselling or education as a stand-alone intervention.

\subsection{Cognitive Outcomes}

Table 2 summarises the cognition assessment tools used in each study. Further details about the cognition assessment tests used is provided in Supplementary Table S5. The age of child cognitive testing ranged from less than two weeks [76] to nine years old [71], however $62 \%$ of studies ( $n=13$ trials) reported cognition outcomes in children less than 35 months, and $46 \%$ of these studies ( $n=6$ trials) provided LCPUFA interventions in high-income countries. Meta-analysis was completed for nine cognition outcomes measured in the 34 included studies. The analyses for each cognition domain is described below, however in summary all cognition domains were not significantly affected by nutritional interventions. 
Table 2. Overview of the characteristics of the randomised controlled trials (RCTs).

\begin{tabular}{|c|c|c|c|c|c|c|c|}
\hline $\begin{array}{l}\text { Source, Year, } \\
\text { Country, OECD }\end{array}$ & $\begin{array}{l}\text { Infant } \\
\text { Sample }(n)\end{array}$ & Intervention Group/s & Control Group/s & $\begin{array}{l}\text { Intervention } \\
\text { Duration }\end{array}$ & $\begin{array}{l}\text { Cognitive } \\
\text { Testing Age }\end{array}$ & $\begin{array}{l}\text { Cognitive } \\
\text { Assessment Tests }\end{array}$ & Main Findings \\
\hline \multicolumn{8}{|c|}{ Choline Intervention vs. Control } \\
\hline $\begin{array}{l}\text { Cheatham et al. [45] } \\
\text { 2012, US, } 1\end{array}$ & 99 & $\begin{array}{l}\text { Supplement: } \\
\text { Vitamins: } 750 \mathrm{mg} / \text { day choline }\end{array}$ & $\begin{array}{l}\text { Supplement: corn } \\
\text { oil capsules }\end{array}$ & $\begin{array}{l}18 \text { weekends gestation } \\
\text { to } 3 \text { months } \\
\text { postnatally }\end{array}$ & 10 and 12 month & $\begin{array}{l}\text { Visuospatial } \\
\text { Memory Delayed } \\
\text { Response } \\
\text { Task [78-80], } \\
\text { Imitation } \\
\text { paradigms [81], } \\
\text { CDI [82] \& } \\
\text { MSEL [83] }\end{array}$ & $\begin{array}{l}\text { There were no significant differences } \\
\text { between the intervention and control } \\
\text { groups for global development, } \\
\text { language, short-term visuospatial } \\
\text { memory, or long-term episodic } \\
\text { memory test scores }(p>0.05) \text { at } 10 \\
\text { and } 12 \text { months }\end{array}$ \\
\hline \multicolumn{8}{|c|}{ Iodine Intervention vs. Control } \\
\hline $\begin{array}{l}\text { Santiago et al. [54], } \\
\text { Spain, } 1\end{array}$ & 102 & $\begin{array}{l}\text { Supplement: } \\
\text { Mineral: I1: } 200 \mu \mathrm{g} / \text { day iodine I2: } \\
300 \mu \mathrm{g} / \text { day iodine }\end{array}$ & $\begin{array}{l}\text { Fortified food: used } \\
\text { iodised salt }\end{array}$ & $\begin{array}{l}\text { Before } 10 \text { weekends } \\
\text { gestation to delivery }\end{array}$ & 6 to 18 month & BSID-III [34] & $\begin{array}{l}\text { There were no significant differences } \\
\text { between the groups for MDI and PDI } \\
\text { scores }(p>0.05) \text { at } 6 \text { to } 18 \text { months }\end{array}$ \\
\hline $\begin{array}{l}\text { Zhou et al. [70], } \\
\text { Australia, } 1\end{array}$ & 53 & $\begin{array}{l}\text { Supplement: } \\
\text { Mineral: } 150 \mu \mathrm{g} / \text { day iodine }\end{array}$ & No supplement & $\begin{array}{l}\text { Less than } 20 \text { weekends } \\
\text { gestation to delivery }\end{array}$ & 18 month & BSID-III [34] & $\begin{array}{l}\text { There was no significant difference } \\
\text { between the intervention compared } \\
\text { to the control group for child } \\
\text { cognition }(p=0.42) \text {, language } \\
(p=0.83) \text { and motor skills }(p=0.61) \\
\text { at } 18 \text { months of age }\end{array}$ \\
\hline $\begin{array}{l}\text { Zhou et al. [65], } \\
\text { Australia, } 1\end{array}$ & 302 & $\begin{array}{l}\text { Supplement: } \\
\text { Mineral: } 20 \mathrm{mg} / \text { day Fe }\end{array}$ & No supplement & $\begin{array}{l}20 \text { weekends gestation } \\
\text { to delivery }\end{array}$ & 48 months & SB4 [84] \& SDQ [85] & $\begin{array}{l}\text { There were no significant differences } \\
\text { between groups for child cognition } \\
\text { and behaviour test scores }(p>0.05) \text { at } \\
48 \text { months }\end{array}$ \\
\hline \multicolumn{8}{|c|}{ Iron and Folic Acid or Multiple Micronutrient Intervention vs. Control } \\
\hline $\begin{array}{l}\text { Chang et al. [73], } \\
\text { China, } 3\end{array}$ & 850 & $\begin{array}{l}\text { Supplement: Vitamins: I1: } \\
400 \mu \mathrm{g} / \text { day FA I2: } 400 \mu \mathrm{g} / \text { day FA, } \\
800 \mu \mathrm{g} / \text { day vit } \mathrm{A}, 1.4 \mathrm{mg} / \text { day vit } \\
\mathrm{B} 1,1.4 \mathrm{mg} / \text { day vit B2, } 18 \mathrm{mg} / \text { day } \\
\mathrm{B} 3,1.9 \mathrm{mg} / \text { day vit B6, } 2.6 \mu \mathrm{g} / \text { day } \\
\text { vit B12, } 70 \mu \mathrm{g} / \text { day vit } \mathrm{C}, 5 \mu \mathrm{g} / \text { day } \\
\text { vit } \mathrm{D}, 10 \mathrm{mg} / \text { day vit } \mathrm{E} \\
\text { Minerals: } \mathrm{I} 1: 60 \mathrm{mg} / \text { day Fe } \mathrm{I} 2: \\
2 \mathrm{mg} / \text { day Cu, } 150 \mu \mathrm{g} / \text { day iodine, } \\
30 \mathrm{mg} / \text { day Fe, } 65 \mu \mathrm{g} / \text { day Se, } \\
15 \mathrm{mg} / \text { day } \mathrm{Zn}\end{array}$ & $\begin{array}{l}\text { Supplement: } \\
\text { Vitamin: } 400 \mu \mathrm{g} / \text { day FA }\end{array}$ & $\begin{array}{l}\text { Less than } 28 \text { weekends } \\
\text { gestation to delivery }\end{array}$ & $\begin{array}{l}3,6,12,18 \\
\text { and } 24 \text { months }\end{array}$ & $\begin{array}{l}\text { BSID-II (Chinese } \\
\text { translation) }[86,87]\end{array}$ & $\begin{array}{l}\text { The prenatal IDA intervention } \\
\text { groups had a lower MDI ( } p=0.046 \text { in } \\
\text { folic acid; } p=0.034 \text { in multiple } \\
\text { micronutrient supplements) } \\
\text { compared to the prenatal-non-IDA } \\
\text { group. There was no significant } \\
\text { difference in MDI scores in the } \\
\text { prenatal-IDA group and } \\
\text { prenatal-non-IDA group that } \\
\text { received iron and folic acid } \\
\text { supplements ( } p=0.641)\end{array}$ \\
\hline
\end{tabular}


Table 2. Cont

\begin{tabular}{|c|c|c|c|c|c|c|c|}
\hline $\begin{array}{l}\text { Source, Year, } \\
\text { Country, OECD }\end{array}$ & $\begin{array}{l}\text { Infant } \\
\text { Sample }(n)\end{array}$ & Intervention Group/s & Control Group/s & $\begin{array}{l}\text { Intervention } \\
\text { Duration }\end{array}$ & $\begin{array}{l}\text { Cognitive } \\
\text { Testing Age }\end{array}$ & $\begin{array}{l}\text { Cognitive } \\
\text { Assessment Tests }\end{array}$ & Main Findings \\
\hline $\begin{array}{l}\text { Li et al. [60], } \\
\text { China, } 2\end{array}$ & 1305 & $\begin{array}{l}\text { Supplement: } \\
\text { Vitamins: I1: } 800 \mu \mathrm{\mu g} / \text { day vit A, } \\
1.4 \mathrm{mg} / \text { day vit B1, } 1.4 \mathrm{mg} / \text { day B2, } \\
18 \mathrm{mg} / \text { day vit B3, } 1.9 \mathrm{mg} / \text { day vit } \\
\mathrm{B} 6,2.6 \mathrm{mg} / \text { day vit B12, } 70 \mathrm{mg} / \text { day } \\
\text { vit C, } 10 \mu \mathrm{g} / \text { day vit D, } 10 \mathrm{mg} / \text { day } \\
\text { vit } \mathrm{E}, 400 \mu \mathrm{g} / \text { day FA I2: } \\
400 \mu \mathrm{g} / \text { day FA Minerals: I1: } \\
2 \mathrm{mg} / \text { day Cu, } 30 \mathrm{mg} / \text { day Fe, } \\
150 \mu \mathrm{gg} / \text { day iodine, } 65 \mu \mathrm{g} / \text { day Se } \\
\mathrm{I} 2: 30 \mathrm{mg} / \text { day Fe }\end{array}$ & $\begin{array}{l}\text { Supplement } \\
\text { Vitamins: } \\
400 \mu \mathrm{g} / \text { day FA }\end{array}$ & $\begin{array}{l}27 \text { weekends gestation } \\
\text { to delivery }\end{array}$ & $\begin{array}{l}3,6 \text { and } \\
12 \text { months }\end{array}$ & BSID-II [86] & $\begin{array}{l}\text { Positive effects were demonstrated } \\
\text { from the multiple micronutrient } \\
\text { supplement on child MDI scores at } \\
12 \text { months }(p=0.01)\end{array}$ \\
\hline $\begin{array}{l}\text { Hanieh et al. [48], } \\
\text { Vietnam, } 3\end{array}$ & 1168 & 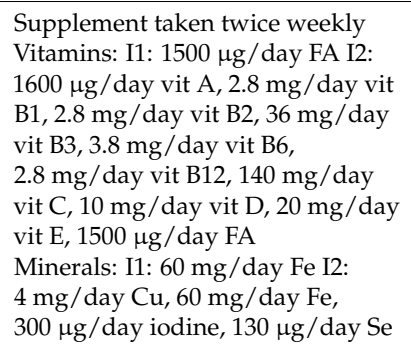 & $\begin{array}{l}\text { Supplement taken daily } \\
\text { Vitamin: } 400 \mathrm{\mu g} / \text { day FA } \\
\text { Mineral: } 60 \mathrm{mg} / \text { day Fe }\end{array}$ & $\begin{array}{l}\text { Less than } 16 \\
\text { weekends gestation to } \\
3 \text { months postnatally }\end{array}$ & 6 months & BSID-III [34] & $\begin{array}{l}\text { A positive effect was demonstrated } \\
\text { from the twice weekly iron and folate } \\
\text { acid supplement groups on child } \\
\text { cognitive development scores } \\
\text { (MD 1.89; } 95 \% \text { CI } 0.23 \text { to } 3.56 \text { ) } \\
\text { at } 6 \text { months }\end{array}$ \\
\hline \multicolumn{8}{|c|}{ LCPUFA s Intervention vs. Control } \\
\hline $\begin{array}{l}\text { Brei et al. [72], } \\
\text { Germany, } 1\end{array}$ & 130 & $\begin{array}{l}\text { Supplement: fish oil capsules } \\
\text { LCPUFAs: } 1020 \mathrm{mg} / \text { day DHA, } \\
180 \mathrm{mg} / \text { day EPA } \\
\text { Vitamin: } 9 \mathrm{mg} / \text { day vit E } \\
\text { Dietary counselling to } \\
\text { lower ARA intake }\end{array}$ & $\begin{array}{l}\text { Participants received } \\
\text { information for eating a } \\
\text { healthy diet } \\
\text { during pregnancy }\end{array}$ & $\begin{array}{l}15 \text { weekends gestation } \\
\text { to } 4 \text { months } \\
\text { postnatally }\end{array}$ & 60 months & $\begin{array}{l}\text { Child Development } \\
\text { Inventory (German } \\
\text { translation) }[88,89] \\
\text { MM }[90,91]\end{array}$ & $\begin{array}{l}\text { There were no differences between } \\
\text { the maternal fish oil supplemented } \\
\text { group compared to the control group } \\
\text { on child CDI scores and MM at } 4 \text { and } \\
5 \text { years of age }(p>0.05)\end{array}$ \\
\hline $\begin{array}{l}\text { Dunstan et al. [46], } \\
\text { Australia, } 1\end{array}$ & 72 & $\begin{array}{l}\text { Supplement: fish oil capsules } \\
\text { LCPUFAs: } 2.2 \mathrm{~g} / \text { day DHA, } \\
1.1 \mathrm{~g} / \text { day EPA } \\
\text { Vitamins: } 3-4 \mathrm{mg} / \text { day vit } \mathrm{E}\end{array}$ & $\begin{array}{l}\text { Supplement: olive oil } \\
\text { capsules } \\
\text { LCPUFA: } 2.7 \mathrm{~g} / \text { day } \\
\text { oleic acid } \\
\text { Vitamins: } 3-4 \mathrm{mg} / \text { day } \\
\text { vit E }\end{array}$ & $\begin{array}{l}20 \text { weekends gestation } \\
\text { to delivery }\end{array}$ & 18 months & $\begin{array}{l}\text { GMDS 0-2 [92], } \\
\text { PPVT-III [93] \& } \\
\text { CBCL [94] }\end{array}$ & $\begin{array}{l}\text { A positive effect was demonstrated } \\
\text { from the maternal fish oil } \\
\text { intervention on eye and hand } \\
\text { coordination }(p=0.021) \text { in children at } \\
18 \text { months }\end{array}$ \\
\hline $\begin{array}{l}\text { Gould et al. [47], } \\
\text { Australia, } 1\end{array}$ & 185 & $\begin{array}{l}\text { Intervention: marine oil capsules } \\
\text { LCPUFAs: } 0.8 \mathrm{~g} / \text { day DHA, } \\
0.1 \mathrm{~g} / \text { day EPA }\end{array}$ & $\begin{array}{l}\text { Supplement: capsule } \\
\text { with a blend of } \\
\text { vegetable oils }\end{array}$ & $\begin{array}{l}\text { 18-21 weekends } \\
\text { gestation to delivery }\end{array}$ & 27 months & $\begin{array}{l}\text { Single and multiple } \\
\text { object task }[95,96] \text { \& } \\
\text { A-not-B task }[97]\end{array}$ & $\begin{array}{l}\text { There were no significant differences } \\
\text { between the intervention and control } \\
\text { groups for child attention, working } \\
\text { memory and inhibitory control } \\
\text { development test scores }(p>0.05)\end{array}$ \\
\hline
\end{tabular}


Table 2. Cont.

\begin{tabular}{|c|c|c|c|c|c|c|c|}
\hline $\begin{array}{l}\text { Source, Year, } \\
\text { Country, OECD }\end{array}$ & $\begin{array}{l}\text { Infant } \\
\text { Sample }(n)\end{array}$ & Intervention Group/s & Control Group/s & $\begin{array}{l}\text { Intervention } \\
\text { Duration }\end{array}$ & $\begin{array}{l}\text { Cognitive } \\
\text { Testing Age }\end{array}$ & $\begin{array}{l}\text { Cognitive } \\
\text { Assessment Tests }\end{array}$ & Main Findings \\
\hline $\begin{array}{l}\text { Helland et al. [49], } \\
\text { Norway, } 1\end{array}$ & 262 & $\begin{array}{l}\text { Supplement: cod liver oil } \\
\text { LCPUFAs: } 75 \mathrm{mg} / \text { day ALA, } \\
27.5 \mathrm{mg} / \text { day ARA, } 1.18 \mathrm{~g} / \text { day } \\
\text { DHA, } 0.8 \mathrm{~g} / \text { day EPA, } 160 \mathrm{mg} / \text { day } \\
\mathrm{LA}, \text { Vitamins: } 117 \mu \mathrm{g} / \text { day vit } \mathrm{A}, \\
1 \mu \mathrm{g} / \text { day vit } \mathrm{D}, 1.4 \mathrm{mg} / \text { day vit } \mathrm{E}\end{array}$ & $\begin{array}{l}\text { Supplement: corn oil } \\
\text { LCPUFAs: } 92 \mathrm{mg} / \text { day } \\
\text { ALA, } 8.3 \mathrm{mg} / \text { day DHA, } \\
4.7 \mathrm{~g} / \text { day LA Vitamins: } \\
117 \mu \mathrm{g} / \text { day vit } \mathrm{A}, \\
1 \mu \mathrm{g} / \text { day vit } \mathrm{D}, \\
1.4 \mathrm{mg} / \text { day vit } \mathrm{E}\end{array}$ & $\begin{array}{l}17-19 \text { weekends } \\
\text { gestation to } 3 \text { months } \\
\text { postnatally }\end{array}$ & $\begin{array}{l}27 \text { and } 39 \\
\text { weekends }\end{array}$ & FTII-II [98] & $\begin{array}{l}\text { There were no significant differences } \\
\text { between the intervention and control } \\
\text { groups for cognitive development } \\
\text { test scores ( } p>0.05 \text { ) at } 27 \text { and } \\
39 \text { months }\end{array}$ \\
\hline $\begin{array}{l}\text { Helland et al. [51], } \\
\text { Norway, } 1\end{array}$ & 90 & $\begin{array}{l}\text { Supplement: cod liver oil } \\
\text { LCPUFAs: } 75 \mathrm{mg} / \text { day ALA, } \\
27.5 \mathrm{mg} / \text { day ARA, } 1.18 \mathrm{~g} / \text { day } \\
\text { DHA, } 0.8 \mathrm{~g} / \text { day EPA, } 160 \mathrm{mg} / \text { day } \\
\mathrm{LA}, \text { Vitamins: } 117 \mu \mathrm{g} / \text { day vit A, } \\
1 \mu \mathrm{g} / \text { day vit } \mathrm{D}, 1.4 \mathrm{mg} / \text { day vit } \mathrm{E}\end{array}$ & 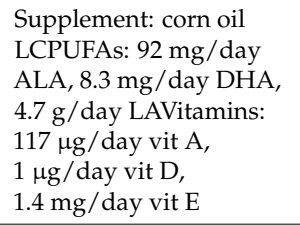 & $\begin{array}{l}1-19 \text { weekends } \\
\text { gestation to } 3 \text { months } \\
\text { postnatally }\end{array}$ & 48 months & KABC-II [99] & $\begin{array}{l}\text { Apositive effect was demonstrated } \\
\text { from the cod liver oil supplement on } \\
\text { child mental processing composite } \\
\text { score }(p=0.049) \text { at } 48 \text { months }\end{array}$ \\
\hline $\begin{array}{l}\text { Helland et al. [50], } \\
\text { Norway, } 1\end{array}$ & 143 & $\begin{array}{l}\text { Supplement: cod liver oil } \\
\text { LCPUFAs: } 75 \mathrm{mg} / \text { day ALA, } \\
27.5 \mathrm{mg} / \text { day ARA, } 1.18 \mathrm{~g} / \text { day } \\
\text { DHA, } 0.8 \mathrm{~g} / \text { day EPA, } 160 \mathrm{mg} / \text { day } \\
\text { LA, Vitamins: } 117 \mu \mathrm{g} / \text { day vit A, } \\
1 \mu \mathrm{g} / \text { day vit } \mathrm{D}, 1.4 \mathrm{mg} / \text { day vit E }\end{array}$ & $\begin{array}{l}\text { Supplement: corn oil } \\
\text { LCPUFAs: } 92 \mathrm{mg} / \text { day } \\
\text { ALA, } 8.3 \mathrm{mg} / \text { day DHA, } \\
4.7 \mathrm{~g} / \text { day LA } \\
\text { Vitamins: } 117 \mu \mathrm{g} / \text { day } \\
\text { vit A, } 1 \mu \mathrm{g} / \text { day vit D, } \\
1.4 \mathrm{mg} / \text { day vit E }\end{array}$ & $\begin{array}{l}1-19 \text { weekends } \\
\text { gestation to } 3 \text { months } \\
\text { postnatally }\end{array}$ & 84 months & KABC-II [99] & $\begin{array}{l}\text { No significant differences between } \\
\text { the intervention and control groups } \\
\text { for cognitive development test scores } \\
(p>0.05) \text { at } 84 \text { months was found }\end{array}$ \\
\hline $\begin{array}{l}\text { Hurtado et al. [68], } \\
\text { Spain, } 1\end{array}$ & 61 & $\begin{array}{l}\text { Supplement: fish oil drink } \\
\text { per } 100 \mathrm{~mL} \\
\text { Energy: } 145 \mathrm{~kJ} \\
\text { Macronutrients: } 3.7 \mathrm{~g} \text { prot., } 6.7 \mathrm{~g} \\
\text { carb., } 1.8 \mathrm{~g} \text { total fat } \\
\text { LCPUFAs: } 80 \mathrm{mg} \text { DHA, } 18 \mathrm{mg} \text { EPA } \\
\text { Vitamins: } 0.4 \mu \mathrm{g} \text { vit B12, } 9 \mathrm{mg} \text { vit C, } \\
0.75 \mu \mathrm{g} \text { vit } \mathrm{D}, 1.5 \mathrm{mg} \text { vit E, } 80 \mu \mathrm{g} \text { FA } \\
\text { Minerals: } 23 \mu \mathrm{g} \text { iodine, } 2.2 \mathrm{mg} \mathrm{Fe}\end{array}$ & $\begin{array}{l}\text { Supplement drink } \\
\text { per } 100 \mathrm{~mL} \\
\text { Energy: } 145 \mathrm{~kJ} \\
\text { Macronutrients: } 3.7 \mathrm{~g} \\
\text { prot., } 6.7 \mathrm{~g} \text { carb., } 1.8 \mathrm{~g} \\
\text { total fat } \\
\text { Vitamins: } 0.4 \mu \mathrm{g} \text { vit B12, } \\
9 \mathrm{mg} \text { vit C, } 0.75 \mu \mathrm{g} \text { vit D, } \\
1.5 \mathrm{mg} \text { vit E, } 80 \mu \mathrm{g} \text { FA } \\
\text { Minerals: } 23 \mu \mathrm{g} \text { iodine, } \\
2.2 \mathrm{mg} \text { Fe }\end{array}$ & $\begin{array}{l}28 \text { weekends gestation } \\
\text { to } 4 \text { months } \\
\text { postnatally }\end{array}$ & 12 months & BSID-II [86] & $\begin{array}{l}\text { There were no differences between } \\
\text { the maternal fish oil supplemented } \\
\text { group compared to the control group } \\
\text { on child MDI and PDI composite } \\
\text { scores at } 12 \text { months of age }\end{array}$ \\
\hline $\begin{array}{l}\text { Judge et al. [59], } \\
\text { US, } 1\end{array}$ & 29 & $\begin{array}{l}\text { Muesli bar with fish oil. Per bar; } \\
\text { Energy: } 386 \text { kJ Macronutrients: } 18 \mathrm{~g} \\
\text { carb., } 0.3 \mathrm{~g} \text { fibre, } 1.3 \mathrm{~g} \text { prot., } 1.7 \mathrm{~g} \\
\text { total fat, } 300 \mathrm{mg} \text { DHA, } 38 \mathrm{mg} \text { EPA }\end{array}$ & $\begin{array}{l}\text { Muesli bar without fish } \\
\text { oil. Per bar; } \\
\text { Energy: } 386 \mathrm{~kJ} \\
\text { Macronutrient: } 18 \mathrm{~g} \\
\text { carb., } 0.3 \mathrm{~g} \text { fibre, } 1.3 \mathrm{~g} \\
\text { prot., } 1.7 \mathrm{~g} \text { total fat }\end{array}$ & $\begin{array}{l}24 \text { weekends gestation } \\
\text { until delivery }\end{array}$ & 9 months & $\begin{array}{l}\text { 2-step } \\
\text { problem-solving } \\
\text { test [100-103] \& } \\
\text { FTII-II [98] }\end{array}$ & $\begin{array}{l}\text { Positive effects were demonstrated } \\
\text { from the fish oil intervention group } \\
\text { on the problem-solving task } \\
(p=0.017) \text {, total intentional score } \\
(p=0.011) \text { and the number of } \\
\text { intentional solution on both cloth } \\
(p=0.008) \text { and cover steps }(p=0.004) \\
\text { at } 9 \text { months }\end{array}$ \\
\hline
\end{tabular}


Table 2. Cont

\begin{tabular}{|c|c|c|c|c|c|c|c|}
\hline $\begin{array}{l}\text { Source, Year, } \\
\text { Country, OECD }\end{array}$ & $\begin{array}{l}\text { Infant } \\
\text { Sample }(n)\end{array}$ & Intervention Group/s & Control Group/s & $\begin{array}{l}\text { Intervention } \\
\text { Duration }\end{array}$ & $\begin{array}{l}\text { Cognitive } \\
\text { Testing Age }\end{array}$ & $\begin{array}{l}\text { Cognitive } \\
\text { Assessment Tests }\end{array}$ & Main Findings \\
\hline $\begin{array}{l}\text { Makrides et al. [52], } \\
\text { Australia, } 1\end{array}$ & 726 & $\begin{array}{l}\text { Supplement: fish oil capsules } \\
\text { LCPUFAs: } 800 \mathrm{mg} \text { /day DHA, } \\
100 \mathrm{mg} / \text { day EPA }\end{array}$ & $\begin{array}{l}\text { Supplement: Capsules } \\
\text { with vegetable oil blend }\end{array}$ & $\begin{array}{l}21 \text { weekends gestation } \\
\text { to delivery }\end{array}$ & 18 months & BSID-III [34] & $\begin{array}{l}\text { There were no significant differences } \\
\text { between the groups for cognitive and } \\
\text { language test scores }(p>0.05)\end{array}$ \\
\hline $\begin{array}{l}\text { Mulder et al. [62], } \\
\text { Canada }{ }^{4}, 1\end{array}$ & 216 & $\begin{array}{l}\text { Supplement: algal oil capsules } \\
\text { LCPUFAs: } 400 \mathrm{mg} / \text { day DHA }\end{array}$ & $\begin{array}{l}\text { Supplement: soybean } \\
\text { and corn oil capsules }\end{array}$ & $\begin{array}{l}16 \text { weekends gestation } \\
\text { to delivery }\end{array}$ & 9 and 18 months & $\begin{array}{l}\text { CDI [82] \& } \\
\text { BSID-III [34] }\end{array}$ & $\begin{array}{l}\text { Negative effects were demonstrated } \\
\text { from the maternal algal oil } \\
\text { supplement for CDI language } \\
(p<0.05) \text { at } 14 \text { months and } \\
18 \text { months and BSID-III language } \\
\text { scores at } 18 \text { months }(p<0.05)\end{array}$ \\
\hline $\begin{array}{l}\text { Ramakrishnan et al. [74 } \\
\text { Mexico, } 2\end{array}$ & & $\begin{array}{l}\text { Supplement: algal oil capsules } \\
\text { LCPUFAs: } 400 \mathrm{mg} / \text { day DHA }\end{array}$ & $\begin{array}{l}\text { Supplement: corn and } \\
\text { soy oil capsules }\end{array}$ & $\begin{array}{l}18-22 \text { weekends } \\
\text { Gestation to delivery }\end{array}$ & 60 months & $\begin{array}{l}\text { MSCA (Spanish } \\
\text { translation) [104], } \\
\text { (BASC-2) [105], and } \\
\text { (K-CPT) [106] }\end{array}$ & $\begin{array}{l}\text { There were no significant differences } \\
\text { between the intervention group } \\
\text { compared to the control group for } \\
\text { MSCA scores }(p>0.05) \text {, BASC-2 or } \\
\text { scores at } 5 \text { years. of age. The } \\
\text { intervention did significantly affect } \\
\text { K-CPT omissions scores }(p=0.01) \\
\text { compared to the placebo group }\end{array}$ \\
\hline $\begin{array}{l}\text { Tofail et al. [63], } \\
\text { Bangladesh, } 4\end{array}$ & 249 & $\begin{array}{l}\text { Supplement: fish oil capsules } \\
\text { LCPUFAs: } 1.2 \mathrm{~g} / \text { day DHA, } \\
1.8 \mathrm{~g} / \text { day EPA }\end{array}$ & $\begin{array}{l}\text { Supplement: soy oil } \\
\text { capsules } \\
\text { LCPUFAs: } 2.3 \mathrm{~g} / \text { day LA }\end{array}$ & $\begin{array}{l}27 \text { weekends gestation } \\
\text { to delivery }\end{array}$ & 10 months & $\begin{array}{l}\text { BSID-II [86] \& } \\
\text { Wolke's Behaviour } \\
\text { Rating Scale [107] }\end{array}$ & $\begin{array}{l}\text { There were no significant differences } \\
\text { between the intervention and control } \\
\text { groups for cognitive or behaviour } \\
\text { development test scores }(p>0.05) \text { at } \\
10 \text { months }\end{array}$ \\
\hline $\begin{array}{l}\text { Van Goor et al. [56], } \\
\text { Netherlands, } 1\end{array}$ & 114 & $\begin{array}{l}\text { Fish oil capsules } \\
\mathrm{I} 1: 220 \mathrm{mg} / \text { day DHA, } 34 \mathrm{mg} / \text { day } \\
\text { EPA, } 15 \mathrm{mg} / \text { day ARA, } 274 \mathrm{mg} / \text { day } \\
\mathrm{LA}, 32 \mathrm{mg} / \text { day ALA I2: } \\
220 \mathrm{mg} \text { /day DHA, } 36 \mathrm{mg} \text { /day EPA, } \\
220 \mathrm{mg} / \text { day ARA, } 46 \mathrm{mg} / \text { day LA, } \\
7 \mathrm{mg} \text { /day ALA }\end{array}$ & $\begin{array}{l}\text { Supplement: soy bean } \\
\text { oil capsules }\end{array}$ & $\begin{array}{l}14-20 \text { weekends } \\
\text { gestation to } 3 \text { months } \\
\text { postnatally }\end{array}$ & 18 months & BSID-II [86] & $\begin{array}{l}\text { There were no significant differences } \\
\text { between the control and intervention } \\
\text { groups for cognitive development } \\
\text { test scores }(p>0.05) \text { at } 18 \text { months }\end{array}$ \\
\hline \multicolumn{8}{|c|}{ LCPUFA and Folic Acid Intervention vs. Control } \\
\hline $\begin{array}{l}\text { Catena et al. [66], } \\
\text { Germany, Spain \& } \\
\text { Hungary, } 1\end{array}$ & 136 & $\begin{array}{l}\text { Dairy drink per sachet: I1: } \\
500 \mathrm{mg} / \text { day DHA, } 150 \mathrm{mg} / \text { day } \\
\text { EPA I2: } 400 \mu \mathrm{\mu g} / \text { day FA I3: } \\
500 \mathrm{mg} / \text { day DHA, } 150 \mathrm{mg} / \text { day } \\
\text { EPA, } 400 \mu \mathrm{\mu g} / \text { day FA }\end{array}$ & $\begin{array}{l}\text { Dairy drink with } \\
\text { vitamins and minerals } \\
\text { in accordance to } \\
\text { European dietary } \\
\text { guidelines for } \\
\text { pregnancy }\end{array}$ & $\begin{array}{l}\text { Less than } 20 \text { weekends } \\
\text { gestation to delivery }\end{array}$ & 102 months & $\begin{array}{l}\text { Attention Network } \\
\text { Test (child } \\
\text { version) }[108,109]\end{array}$ & $\begin{array}{l}\text { Children born to mothers } \\
\text { supplemented with folic acid } \\
\text { alone solved the response conflict } \\
\text { more quickly than the control group } \\
\text { and the fish oiand folic acid group } \\
\text { (all } p<0.05 \text { ) }\end{array}$ \\
\hline
\end{tabular}


Table 2. Cont.

\begin{tabular}{|c|c|c|c|c|c|c|c|}
\hline $\begin{array}{l}\text { Source, Year, } \\
\text { Country, OECD }\end{array}$ & $\begin{array}{l}\text { Infant } \\
\text { Sample }(n)\end{array}$ & Intervention Group/s & Control Group/s & $\begin{array}{l}\text { Intervention } \\
\text { Duration }\end{array}$ & $\begin{array}{l}\text { Cognitive } \\
\text { Testing Age }\end{array}$ & $\begin{array}{l}\text { Cognitive } \\
\text { Assessment Tests }\end{array}$ & Main Findings \\
\hline \multicolumn{8}{|c|}{ Multiple Micronutrient Intervention vs. Control } \\
\hline $\begin{array}{l}\text { Christian et al. [71], } \\
\text { Nepal, } 4\end{array}$ & 676 & $\begin{array}{l}\text { Supplement: } \\
\text { Vitamins: I1: } 1000 \mu \mathrm{g} / \text { day vit A, } \\
400 \mu \mathrm{g} / \text { day FA I2: } 1000 \mu \mathrm{g} / \text { day vit } \\
\mathrm{A}, 400 \mu \mathrm{g} / \text { day FA I3: } 1000 \mu \mathrm{g} / \text { day } \\
\text { vit A, } 400 \mu \mathrm{g} / \text { day FA I4: } \\
1000 \mu \mathrm{g} / \text { day vit A, } 400 \mu \mathrm{g} / \text { day FA, } \\
1.6 \mathrm{mg} / \text { day vit B1, } 1.8 \mathrm{mg} / \text { day vit } \\
\mathrm{B} 2,20 \mathrm{mg} / \mathrm{day} \text { vit B3, } 2.2 \mu \mathrm{g} / \text { day } \\
\text { vit B6, } 2.6 \mu \mathrm{g} / \text { day vit B12, } \\
100 \mathrm{mg} / \text { day vit C, } 10 \mu \mathrm{dg} / \text { day vit D, } \\
10 \mathrm{mg} / \text { day vit E, } 65 \mu \mathrm{g} / \text { day vit K } \\
\text { Minerals: } \mathrm{I2}: 60 \mathrm{mg} / \text { day Fe I3: } \\
60 \mathrm{mg} / \text { day Fe, } 30 \mathrm{mg} / \text { day Zn I4: } \\
2 \mathrm{mg} / \text { day Cu, } 60 \mathrm{mg} / \text { day Fe, } \\
100 \mathrm{mg} / \mathrm{day} \mathrm{Mg}, 30 \mathrm{mg} / \text { day } \mathrm{Zn}\end{array}$ & $\begin{array}{l}\text { Supplement: Vitamin: } \\
1000 \mu \mathrm{g} / \text { day vit A }\end{array}$ & $\begin{array}{l}\text { Pregnancy to } \\
3 \text { months postnatally }\end{array}$ & $84-108$ months & $\begin{array}{l}\text { UNIT [110], } \\
\text { Go/no-go [111], the } \\
\text { Stroop test [112], } \\
\text { backward digit } \\
\text { span [113], } \\
\text { MABC [114], } \\
\text { finger-tapping } \\
\text { test [115] }\end{array}$ & $\begin{array}{l}\text { The difference across all outcomes } \\
\text { (UNIT, executive function and motor } \\
\text { functioning) was significant in } \\
\text { children whose mother received iron } \\
\text { and folic acid supplementation } \\
\text { ( } p=0.001 \text { ) compared to the control } \\
\text { group but not for the other } \\
\text { intervention groups }(p>0.05)\end{array}$ \\
\hline $\begin{array}{l}\text { Christian et al. [67], } \\
\text { Bangladeshi, } 4\end{array}$ & 734 & $\begin{array}{l}\text { Supplement: } \\
\text { Vitamins: } 770 \mu \mathrm{g} / \text { day vit A, } \\
1.4 \mathrm{mg} / \text { day vit B1, } 1.4 \mathrm{mg} / \text { day vit } \\
\mathrm{B} 2,18 \mathrm{mg} / \text { day vit } \mathrm{B} 3,1.9 \mathrm{mg} / \text { day } \\
\text { vit B6, } 2.6 \mu \mathrm{g} / \text { day vit B12, } \\
85 \mathrm{mg} / \text { day vit C, } 5 \mu \mathrm{g} / \text { day vit D, } \\
15 \mathrm{mg} / \text { day vit E, } 600 \mu \mathrm{g} / \text { day FA } \\
\text { Minerals: } 1000 \mu \mathrm{g} / \text { day } \\
\mathrm{Cu}, 220 \mu \mathrm{g} / \text { day iodine, } 27 \mathrm{mg} / \text { day } \\
\mathrm{Fe}, 60 \mu \mathrm{g} \mathrm{Se}, 12 \mathrm{mg} / \text { day } \mathrm{Zn}\end{array}$ & 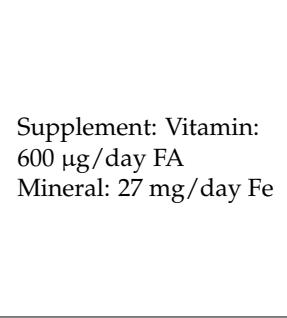 & $\begin{array}{l}\text { Pregnancy to } \\
3 \text { months postnatally }\end{array}$ & 24 months & BSID-III [34] & $\begin{array}{l}\text { There were no differences between } \\
\text { the intervention and control group } \\
\text { on child composite scores for } \\
\text { cognition }(p=0.52) \text {, language } \\
(p=0.97) \text {, and motor performance } \\
(p=0.22) \text { at } 24 \text { months of age }\end{array}$ \\
\hline $\begin{array}{l}\text { Joos et al. [116], } \\
\text { Taiwan, } 2\end{array}$ & 198 & $\begin{array}{l}\text { Supplement: } 1680 \mathrm{~kJ}, \\
50 \mathrm{~g} / \text { day carb., } 0.55 \mathrm{~g} \text { /day fibre, } \\
20 \mathrm{~g} / \text { day prot., } 13.3 \mathrm{~g} / \text { day total } \\
\text { fatVitamins: } 750 \mu \mathrm{g} / \text { day vit } \mathrm{A}, \\
0.8 \mathrm{mg} / \text { day vit } \mathrm{B} 1,0.9 \mathrm{mg} / \text { day vit } \\
\mathrm{B} 2,10 \mathrm{mg} / \text { day vit B3, } 0.08 \mathrm{mg} / \text { day } \\
\text { vit } \mathrm{B} 6,1 \mathrm{mg} / \text { day vit } \mathrm{B} 12, \\
37.5 \mathrm{mg} / \text { day vit } \mathrm{C}, 5 \mathrm{\mu g} / \text { day vit } \mathrm{D} \text {, } \\
3.35 \mathrm{mg} / \text { day vit } \mathrm{E} \text { Minerals: } \\
0.5 \mathrm{mg} / \text { day Ca, } 0.5 \mathrm{mg} / \text { day Cu, } \\
6 \mathrm{mg} / \text { day Fe, } 1 \mathrm{mg} / \text { day Mn, } \\
0.9 \mathrm{mg} / \text { day K, } 0.2 \mathrm{mg} / \text { day Na, } \\
0.4 \mathrm{mg} / \text { day }\end{array}$ & No supplement & $\begin{array}{l}\text { Conception to } \\
\text { lactation of 2nd child }\end{array}$ & 8 months & BSID-I [117] & $\begin{array}{l}\text { A positive effect was demonstrated } \\
\text { from the multiple micronutrient } \\
\text { supplement on PDI (mean } \pm \text { SD: } \\
3.80 \pm 1.90 \text { compared to } 3.31 \pm 1.71) \\
\text { at } 8 \text { months }\end{array}$ \\
\hline
\end{tabular}


Table 2. Cont.

\begin{tabular}{|c|c|c|c|c|c|c|c|}
\hline $\begin{array}{l}\text { Source, Year, } \\
\text { Country, OECD }\end{array}$ & $\begin{array}{l}\text { Infant } \\
\text { Sample }(n)\end{array}$ & Intervention Group/s & Control Group/s & $\begin{array}{l}\text { Intervention } \\
\text { Duration }\end{array}$ & $\begin{array}{l}\text { Cognitive } \\
\text { Testing Age }\end{array}$ & $\begin{array}{l}\text { Cognitive } \\
\text { Assessment Tests }\end{array}$ & Main Findings \\
\hline $\begin{array}{l}\text { Prado et al. [53], } \\
\text { Indonesia, } 3\end{array}$ & 487 & $\begin{array}{l}\text { Supplement } \\
\text { Vitamins: } 800 \mu \mathrm{g} / \text { day vit } \mathrm{A} \text {, } \\
1.4 \mathrm{mg} / \text { day vit B1, } 1.4 \mathrm{mg} / \text { day vit } \\
\mathrm{B} 2,18 \mathrm{mg} / \text { day vit B3, } 1.9 \mathrm{mg} / \text { day } \\
\text { vit B6, } 2.6 \mathrm{mg} / \text { day vit B12, } \\
70 \mathrm{mg} / \text { day vit C, } 5 \mu \mathrm{g} / \text { day vit } \mathrm{D} \text {, } \\
100 \mathrm{mg} \text { vit E, } 400 \mu \mathrm{g} / \text { day FA } \\
\text { Minerals: } 2 \mu \mathrm{g} / \text { day Cu, } 30 \mathrm{mg} / \text { day } \\
\mathrm{Fe}, 150 \mu \mathrm{\mu g} / \text { day iodine, } \\
65 \mu \mathrm{g} / \text { day Se }\end{array}$ & $\begin{array}{l}\text { Supplement: } \\
\text { Vitamins: } \\
400 \mu \mathrm{g} / \text { day FA } \\
\text { Minerals: } 30 \mathrm{mg} / \text { day Fe }\end{array}$ & $\begin{array}{l}\text { Pregnancy to } \\
3 \text { months postnatally }\end{array}$ & 42 months & $\begin{array}{l}\text { BSID-II [86], } \\
\text { ASQ [118], } \\
\text { BPVS-II [119], } \\
\text { BAS-2 [120], } \\
\text { CDI [82], } \\
\text { NEPSY [121], Snack } \\
\text { Delay Test [122,123], } \\
\text { Windows Test [124] } \\
\text { \& Socioemotional } \\
\text { Development } \\
\text { Scale [53]. }\end{array}$ & $\begin{array}{l}\text { No significant differences between } \\
\text { the intervention and control groups } \\
\text { for child motor, language, visual } \\
\text { attention/spatial, } \\
\text { executive, and socio-emotional } \\
\text { development test scores ( } p>0.05 \text { ) at } \\
42 \text { months was found }\end{array}$ \\
\hline $\begin{array}{l}\text { Tofail et al. [64], } \\
\text { Bangladesh, } 4\end{array}$ & 2853 & $\begin{array}{l}\text { Food \& supplements } \\
\text { Energy: } 2512 \mathrm{~kJ} \text {. Vitamins: I1: } 400 \\
\mu \mathrm{g} \text { folate } \mathrm{I}: 1.4 \mathrm{mg} / \text { day vit B1, } \\
1.4 \mathrm{mg} / \text { day vit B2, } 18 \mathrm{mg} / \text { day vit } \\
\mathrm{B} 3,2.6 \mathrm{mg} / \text { day vit B6, } 1.9 \mathrm{mg} / \text { day } \\
\text { vit B12, } 70 \mathrm{mg} / \text { day vit C, } 5 \mu \mathrm{\mu g} / \text { day } \\
\text { vit D, } 10 \mathrm{mg} / \text { day vit E, } \\
400 \mathrm{\mu g} / \text { day FA } \\
\text { Minerals: } \mathrm{I} 1: 30 \mathrm{mg} / \text { day Fe } \mathrm{I} 2: \\
2 \mathrm{mg} / \text { day Cu, } 60 \mathrm{mg} / \text { day Fe, } \\
150 \mu \mathrm{\mu g} / \text { day iodine, } 65 \mu \mathrm{gg} / \text { day, Se } \\
15 \mathrm{mg} / \text { day } \mathrm{Zn}\end{array}$ & 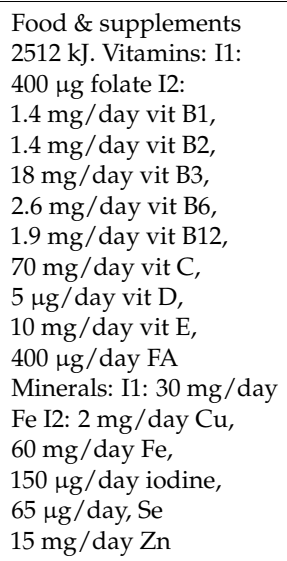 & $\begin{array}{l}17 \text { weekends gestation } \\
\text { to delivery }\end{array}$ & 7 months & $\begin{array}{l}\text { Two step } \\
\text { problem-solving } \\
\text { tests [100-103], } \\
\text { BSID-II [86] \& } \\
\text { Wolke's Behaviour } \\
\text { Rating Scale [107] }\end{array}$ & $\begin{array}{l}\text { There were no significant differences } \\
\text { between the intervention and control } \\
\text { groups for child problem solving } \\
\text { ability, cognitive or behaviour } \\
\text { development test scores }(p>0.05) \text { at } \\
7 \text { months }\end{array}$ \\
\hline $\begin{array}{l}\text { Vuori et al. [76], } \\
\text { Colombia, 2 }\end{array}$ & 244 & $\begin{array}{l}\text { Food \& supplements: } \\
\text { Energy: } 3595 \mathrm{~kJ} \\
\text { Prot: } 38.4 \mathrm{~g} / \text { day } \\
\text { Vitamin: } 1807 \mu \mathrm{\mu g} / \text { day vit A } \\
\text { Mineral: } 18 \mathrm{mg} / \text { day Fe }\end{array}$ & No supplement & $\begin{array}{l}27 \text { weekends gestation } \\
\text { and continued } \\
\text { during lactation }\end{array}$ & 15 days & $\begin{array}{l}\text { Checkboard } \\
\text { activity [76] }\end{array}$ & $\begin{array}{l}\text { Positive effects were demonstrated } \\
\text { from food supplements on infants } \\
\text { that achieved habituation }(70 \%) \text { in } \\
\text { comparison to the control group } \\
(56 \%) p<0.05 \text {. In addition the } \\
\text { supplemented infants moved less } \\
\text { during testing (mean }=11.80, \\
\mathrm{SD}=6.85 \text { ) in comparison to the } \\
\text { supplemented group (mean }=10.19 \text {, } \\
\mathrm{SD}=5.53 \text { ) }\end{array}$ \\
\hline
\end{tabular}


Table 2. Cont.

\begin{tabular}{|c|c|c|c|c|c|c|c|}
\hline $\begin{array}{l}\text { Source, Year, } \\
\text { Country, OECD }\end{array}$ & $\begin{array}{l}\text { Infant } \\
\text { Sample }(n)\end{array}$ & Intervention Group/s & Control Group/s & $\begin{array}{l}\text { Intervention } \\
\text { Duration }\end{array}$ & $\begin{array}{l}\text { Cognitive } \\
\text { Testing Age }\end{array}$ & $\begin{array}{l}\text { Cognitive } \\
\text { Assessment Tests }\end{array}$ & Main Findings \\
\hline $\begin{array}{l}\text { Waber et al. [125], } \\
\text { Colombia, } 2\end{array}$ & 304 & $\begin{array}{l}\text { Food, supplements \& nutrition } \\
\text { education } \\
3595 \mathrm{~kJ} \text { energy, } 38.4 \mathrm{~g} \text { prot., } 25.8 \mathrm{~g} \\
\text { total fat. Vitamins: } 1807 \mu \mathrm{gg} \text { vit A, } \\
5.4 \mathrm{mg} \text { vit B1, } 6.6 \mathrm{mg} \text { vit B2, } \\
31.6 \mathrm{mg} \text { vit B3, } 104.6 \mathrm{mg} \text { vit C } \\
\text { Mineral: } 1146 \mathrm{mg} \mathrm{Ca}\end{array}$ & No supplement & $\begin{array}{l}27 \text { weekends gestation } \\
\text { to } 6 \text { months } \\
\text { postnatally }\end{array}$ & $\begin{array}{l}4,6,12,18,24 \\
\text { and } 36 \text { months }\end{array}$ & $\begin{array}{l}\text { GMDS 0-2 [92] \& } \\
\text { Escalona \& } \\
\text { Corman's Albert } \\
\text { Einstein Scales of } \\
\text { Sensorimotor } \\
\text { Development [126] }\end{array}$ & $\begin{array}{l}\text { Positive effects were demonstrated } \\
\text { from food supplements on subtests } \\
\text { including personal social }(p=0.006) \text {, } \\
\text { speech and language }(p=0.019) \text {, eye } \\
\text { and hand coordination }(p=0.008) \text {, as } \\
\text { well as general quotient }(p=0.003)\end{array}$ \\
\hline \multicolumn{8}{|c|}{ Multiple Micronutrient or Vitamin A Intervention vs. Control } \\
\hline $\begin{array}{l}\text { McGrath et al. [61], } \\
\text { Tanzania, } 4\end{array}$ & 327 & $\begin{array}{l}\text { Supplement: } \\
\text { Vitamins: I1: } 1500 \mu \mathrm{\mu g} / \text { day vit A, } \\
30 \mathrm{mg} / \text { day beta-carotene, } \\
20 \mathrm{mg} / \text { day vit } \mathrm{B} 1,20 \mathrm{mg} / \text { day vit } \\
\mathrm{B} 2,100 \mathrm{mg} / \text { day vit } \mathrm{B} 3,25 \mathrm{mg} / \text { day } \\
\text { vit B6, } 50 \mathrm{mg} / \text { day vit B12, } \\
500 \mathrm{mg} / \text { day vit C, } 30 \mathrm{mg} / \text { day vit E, } \\
800 \mu \mathrm{gg} / \text { day FA } \mathrm{I} 2: 1500 \mu \mathrm{\mu g} \text { day vit } \\
\mathrm{A}, 30 \mathrm{mg} / \text { day beta-carotene, } \\
20 \mathrm{mg} / \text { day }\end{array}$ & $\begin{array}{l}\text { Supplement: } \\
\text { Vitamins: C1: } \\
20 \mathrm{mg} / \text { day vit B1, } \\
20 \mathrm{mg} / \text { day vit B2, } \\
100 \mathrm{mg} \text { /day vit B3, } \\
25 \mathrm{mg} / \text { day vit B6, } \\
50 \mathrm{mg} / \text { day vit B12, } \\
500 \mathrm{mg} / \text { day vit C, } \\
30 \mathrm{mg} / \text { day vit } \mathrm{E}, \\
800 \mathrm{\mu g} / \text { day FA C2: } \\
\text { No supplements }\end{array}$ & $\begin{array}{l}12-27 \text { weekends } \\
\text { gestation to } 18 \text { months } \\
\text { in postnatal period }\end{array}$ & $\begin{array}{l}6,12 \text { and } \\
18 \text { months }\end{array}$ & BSID-II [86] & $\begin{array}{l}\text { Positive effects were demonstrated } \\
\text { from the multiple micronutrient } \\
\text { supplement by increasing PDI score } \\
\text { by } 2.6 \text { points ( } 95 \% \text { CI: } 0.1-5.1 \text { ) over } 6 \\
\text { to } 18 \text { months Vitamin A } \\
\text { supplementation was also associated } \\
\text { with } 2.8 \text { point increase }(95 \% \text { CI: } \\
0.4-5.2 \text { ) in PDI score at } 6 \text { months }\end{array}$ \\
\hline \multicolumn{8}{|c|}{ Vitamin A, Folic Acid and Iron Intervention vs. Control } \\
\hline $\begin{array}{l}\text { Schmidt et al. [69], } \\
\text { Indonesia, } 4\end{array}$ & 188 & $\begin{array}{l}\text { Supplement: } \\
\text { Vitamins: } 4800 \mu \mathrm{g} / \text { day vit A, } \\
500 \mu \mathrm{g} / \text { day FA } \\
\text { Mineral: } 120 \mathrm{mg} / \text { day Fe }\end{array}$ & 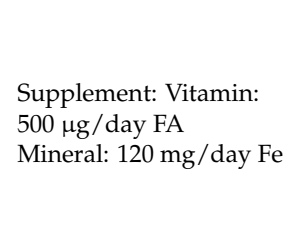 & $\begin{array}{l}16-20 \text { weekends } \\
\text { gestation to delivery }\end{array}$ & 6 and 12 months & BSID-I [117] & $\begin{array}{l}\text { There were no significant effects of } \\
\text { vitamin A supplementation on MDI } \\
\text { and PDI scores at } 6 \text { or } 12 \text { months of } \\
\text { age }(p>0.05) \text {. There was no } \\
\text { significant difference between weekly } \\
\text { compared to daily iron } \\
\text { supplementation on child MDI and } \\
\text { PDI scores }(p>0.05)\end{array}$ \\
\hline \multicolumn{8}{|c|}{ Vitamin B12 Intervention vs. Control } \\
\hline $\begin{array}{l}\text { Srinivasan et al. [75], } \\
\text { India, } 3\end{array}$ & 178 & $\begin{array}{l}\text { Supplement: Vitamin: } 50 \mu \mathrm{g} / \text { day } \\
\text { vit B12 }\end{array}$ & No supplement & $\begin{array}{l}\text { Less than } 14 \text { weekends } \\
\text { gestation to } 6 \\
\text { weekends postnatally }\end{array}$ & 9 months & BSID-III [34] & $\begin{array}{l}\text { There were no significant effects of } \\
\text { maternal B12 supplementation on } \\
\text { child MDI and PDI scores at } \\
9 \text { months }(p>0.05)\end{array}$ \\
\hline \multicolumn{8}{|c|}{ Zinc Intervention vs. Control } \\
\hline $\begin{array}{l}\text { Hamadani et al. [58], } \\
\text { Bangladesh, } 4\end{array}$ & 168 & $\begin{array}{l}\text { Supplement: } \\
\text { Vitamin: } 30 \mathrm{mg} / \text { day Zn }\end{array}$ & No supplement & Pregnancy to delivery & 13 months & $\begin{array}{l}\text { BSID-II [86] \& } \\
\text { Wolke's Behaviour } \\
\text { Rating Scale [107] }\end{array}$ & $\begin{array}{l}\text { Negative effects were demonstrated } \\
\text { from maternal zinc supplementation } \\
\text { on child MDI }(p=0.04) \text { and } \\
\text { psychomotor development index } \\
\text { (PDI) }(p=0.04) \text { at } 13 \text { months }\end{array}$ \\
\hline
\end{tabular}


Table 2. Cont.

\begin{tabular}{|c|c|c|c|c|c|c|c|}
\hline $\begin{array}{l}\text { Source, Year, } \\
\text { Country, OECD }\end{array}$ & $\begin{array}{l}\text { Infant } \\
\text { Sample }(n)\end{array}$ & Intervention Group/s & Control Group/s & $\begin{array}{l}\text { Intervention } \\
\text { Duration }\end{array}$ & $\begin{array}{l}\text { Cognitive } \\
\text { Testing Age }\end{array}$ & $\begin{array}{l}\text { Cognitive } \\
\text { Assessment Tests }\end{array}$ & Main Findings \\
\hline $\begin{array}{l}\text { Tamura et al. [55], } \\
\text { US, } 1\end{array}$ & 347 & $\begin{array}{l}\text { Supplement: } \\
\text { Mineral: } 25 \text { mg/day Zn }\end{array}$ & No supplement & $\begin{array}{l}19 \text { weekends gestation } \\
\text { to delivery }\end{array}$ & 60 months & $\begin{array}{l}\text { DAS [127], } \\
\text { VSM [128], } \\
\text { ASM [128], Knox } \\
\text { Cube [129], } \\
\text { PDMS-2 [130] \& } \\
\text { Grooved } \\
\text { Pegboard [131] }\end{array}$ & $\begin{array}{l}\text { There were no significant differences } \\
\text { between the intervention and control } \\
\text { groups for cognitive, memory or } \\
\text { motor development test scores } \\
(p>0.05) \text { at } 60 \text { months }\end{array}$ \\
\hline \multicolumn{8}{|c|}{ Zinc, Folic Acid and Iron vs. Control } \\
\hline $\begin{array}{l}\text { Caulfield et al. [57], } \\
\text { Peru, } 2\end{array}$ & 184 & $\begin{array}{l}\text { Supplement: } \\
\text { Vitamins: } 250 \mu \mathrm{gg} \text { day FA } \\
\text { Minerals: } 25 \mathrm{mg} / \text { day Zn, } \\
60 \mathrm{mg} / \text { day Fe }\end{array}$ & $\begin{array}{l}\text { Supplement: } \\
\text { Vitamins: } \\
250 \mu \mathrm{g} / \text { day FA } \\
\text { Minerals: } 60 \mathrm{mg} / \text { day Fe }\end{array}$ & $\begin{array}{l}10-14 \text { weekends } \\
\text { gestation to delivery }\end{array}$ & 54 months & $\begin{array}{l}\text { WPPSI-III [113], bear } \\
\text { story [132,133], the } \\
\text { counting game [57], } \\
\text { draw a person [134], } \\
\text { the friendship } \\
\text { interview [135,136], } \\
\text { Vinelands II [97] \& } \\
\text { PBQ [137] }\end{array}$ & $\begin{array}{l}\text { There were no significant differences } \\
\text { between the intervention and control } \\
\text { groups for child cognitive, social or } \\
\text { behavioural development test scores } \\
(p>0.05) \text { at } 54 \text { moths }\end{array}$ \\
\hline
\end{tabular}

ALA: alpha-linolenic acid; ARA: arachidonic acid; ASQ: Ages and Stages Questionnaire, second edition; ASM: Auditory Sequential Memory; BAS-II: British Ability Scales, second edition; BASC-2: Behavioural Assessment System for Children; BPVS: British Picture Vocabulary Scale, second edition; BSID-I: Bayley Scales of Infant Development, first edition; BSID-II: Bayley Scales of Infant Development, second edition; BSID-III: Bayley Scales of Infant Development, third edition; C1: control 1; C2: control 2, Ca: calcium; CBCL: Child Behaviour Checklist; Carb: carbohydrates; CDI: MacArthur-Bates Communicative Development Inventories; CI; confidence interval; CL: confidence level; Cu: copper; DAS: Differential Ability Scales, first edition; DHA: docosahexaenoic acid; EPA: eicosapentaenoic acid; FA: folic acid; Fe: iron; I1: intervention 1; FTII-II: Fagan Test of Infant Intelligence, second edition; I2: intervention 2; I3: intervention 3; I4: intervention 4; IDA: iron deficiency anaemia; GMDS 0-2: Griffiths Mental Development Scales for ages 0-2 years; K: potassium; KABC-II: Kaufman Assessment Battery for Children, second edition; K-CPT: Conners' Kiddie Continuous Performance Test; LA: linoleic acid; LCPUFA: long chain polyunsaturated fatty acids; MABC: Movement Assessment Battery for Children; MD: mean difference; MDI: motor development index; MM: lower mirror movements; Mn: manganese; MSCA: McCarthy Scales of Children's Abilities; MSEL: Mullen Scales of Early Learning; Na: sodium; NEPSY-II: A Developmental Neuropsychological Assessment, second edition; OR: odds ratio; P: phosphorus; PDI: psychomotor development index; PBQ: Preschool Behaviour Questionnaire; PDMS-2: Peabody Developmental Motor Scales, 2nd edition; PPVT-III: Peabody Picture Vocabulary Test, third edition; Prot: protein; SB4: The Stanford-Binet Intelligence Test; SD: standard deviation; DQ: Strength and Difficulties Questionnaire; Se: selenium; SE: standard error; UNIT: Universal Non-verbal
Intelligence Test; Vineland II: Vineland's Adaptive Behaviour Scales, second edition; VSM: Visual Sequential Memory; Vit: vitamin; WPPSI-III: Wechsler Preschool Primary Scale of Intelligence Test; Vineland II: Vineland's Adaptive Behaviour Scales, second edition; VSM: Visual Sequential Memory; Vit: vitamin; WPPSI-III: Wechsler Preschool Primary Scale of
Intelligence, third edition; Zn: zinc. OECD, the organisation for economic co-operation and development criteria: $1=$ high income country, $2=$ higher middle income country, $3=$ lower middle income country \& $4=$ low income country. 


\subsection{Outcome 1: Behaviour}

Ten RCTs $[46,48,52,53,57,64,65,70,72,74]$ measured the effect of a nutritional intervention on behaviour composite scores. Multiple outcomes were combined to create a composite score in Brei et al. [72] (2 outcomes), Makrides et al. [52] (2 outcomes), Ramakrishnan et al. [74] (4 outcomes), Zhou et al. [65] (5 outcomes), Zhou et al. [70] (2 outcomes), Caulfield et al. [57] (5 outcomes), and Dunstan et al. [46] (4 outcomes). There was no significant effect of LCPUFA supplementation ( $n=4$ trials) $[46,52,72,74]$, multiple micronutrient supplementation $(n=1$ trial) [53], iron supplementation ( $n=1$ trial) [65], iodine supplementation ( $n=1$ trial) [70], zinc supplementation ( $n=1$ trial) [57] or a food-based intervention ( $n=1$ trial) [64] (Table 3) (Table 3 and Figure 2). Hanieh et al. [48] investigated the effect of nutrient supplements of varying dosage on cognition, no significant effect on infant behaviour composite score was reported. From the statistical tests of Egger $(p=0.72)$, there was no evidence of publication bias (Supplementary Figure S2). From the sensitivity analysis, the country-income of the studies did not significantly $(p>0.05)$ affect child behaviour outcomes in studies that provided LCPUFA supplementation (Supplementary Figure S3).

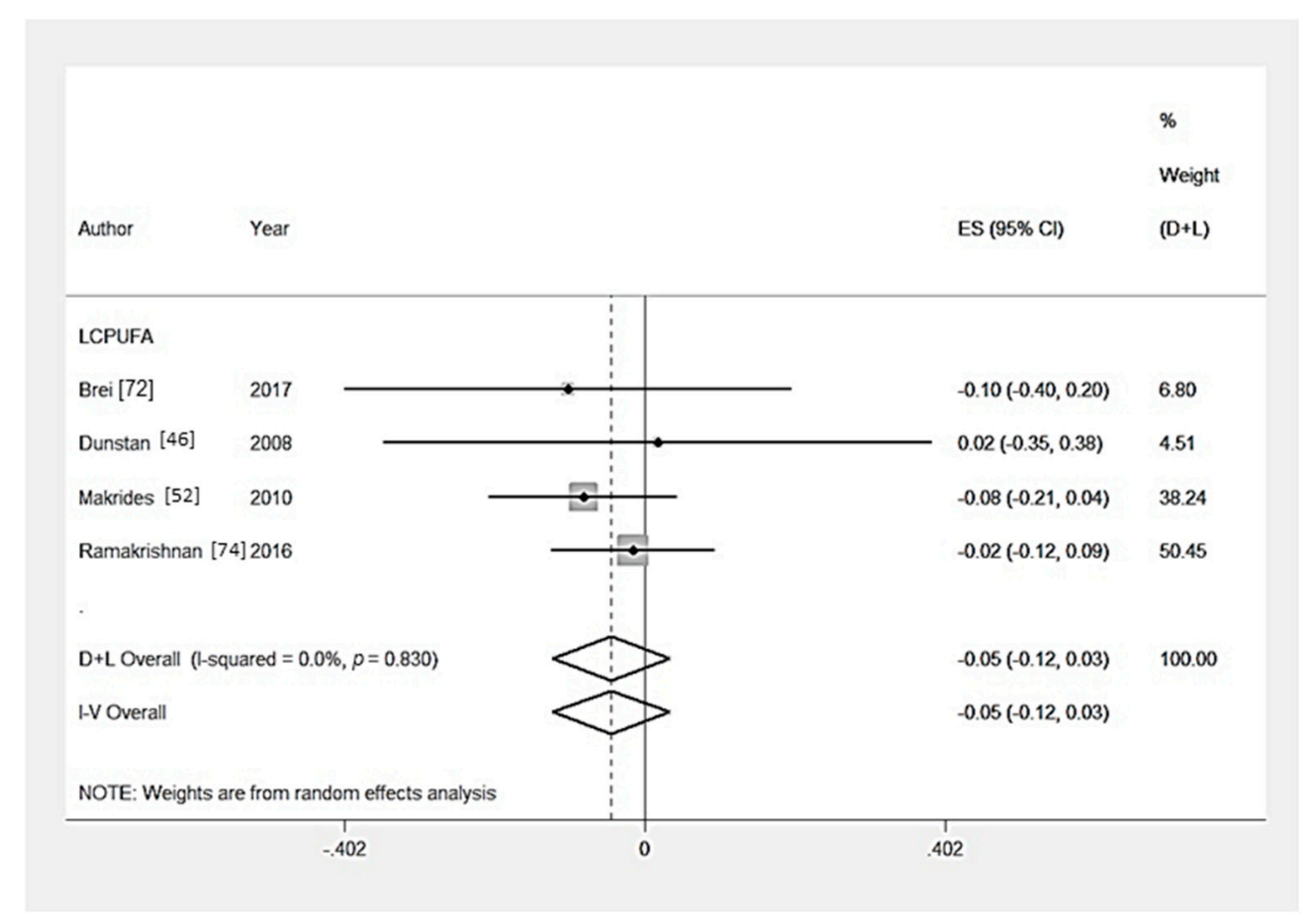

Figure 2. Forest plot for child behaviour outcomes. The overall effect size was estimated by standardised mean difference (SMD). LCPUFA, long chain polyunsaturated fatty acids. $\mathrm{D}+\mathrm{L}$, random-effects estimate (Der Simonian and Laird method) and I-V, fixed-effects estimate (inverse variance method). 
Table 3. Meta-analyses for cognitive outcomes in evaluation of nutritional interventions during pregnancy.

\begin{tabular}{|c|c|c|c|c|c|c|c|c|}
\hline Dietary Nutrient & Cognitive Outcome & Study Authors & Country (OECD) & Studies $(n)$ & Children $(n)$ & SMD $(95 \% \text { CI })^{\dagger}$ & $p$-Value & $I^{2 \ddagger}$ \\
\hline LCPUFA & Attention & $\begin{array}{c}\text { Gould et al. [47] } \\
\text { Ramakrishnan et al. [74] }\end{array}$ & $\begin{array}{l}\text { Australia (1) } \\
\text { Mexico (2) }\end{array}$ & 2 & 955 & $-0.07(-0.17$ to 0.03$)$ & 0.19 & $0 \%$ \\
\hline LCPUFA & Behaviour & $\begin{array}{c}\text { Dunstan et al. [46] } \\
\text { Makrides et al. [52] } \\
\text { Brei et al. [72] } \\
\text { Ramakrishnan et al. [74] }\end{array}$ & $\begin{array}{l}\text { Australia (1) } \\
\text { Australia (1) } \\
\text { Germany (1) } \\
\text { Mexico (2) }\end{array}$ & 4 & 1725 & $-0.05(-0.12$ to 0.03$)$ & 0.25 & $0 \%$ \\
\hline $\mathrm{MMN}$ & Motor skills & $\begin{array}{c}\text { Li et al. [60] } \\
\text { McGrath et al. [61] } \\
\text { Prado et al. [53] } \\
\text { Chang et al. [73] } \\
\text { Christian [71] } \\
\text { Christian [67] }\end{array}$ & $\begin{array}{c}\text { China (2) } \\
\text { Tanzania (4) } \\
\text { Indonesia (3) } \\
\text { China (3) } \\
\text { Nepal (4) } \\
\text { Bangladesh (4) }\end{array}$ & 6 & 3572 & $0.02(-0.04$ to 0.17$)$ & 0.55 & $0 \%$ \\
\hline LCPUFA & Motor skills & $\begin{array}{c}\text { Dunstan et al. [46] } \\
\text { Makrides et al. [52] } \\
\text { Mulder et al. [62] } \\
\text { Tofail et al. [63] } \\
\text { Van Goor et al. [56] } \\
\text { Brei et al. [72] } \\
\text { Ramakrishnan et al. [74] }\end{array}$ & $\begin{array}{c}\text { Australia (1) } \\
\text { Australia (1) } \\
\text { Canada (1) } \\
\text { Bangladesh (4) } \\
\text { Netherlands (1) } \\
\text { Germany (1) } \\
\text { Mexico (2) }\end{array}$ & 7 & 2265 & $0.06(-0.03$ to 0.15$)$ & 0.22 & $8.9 \%$ \\
\hline Zinc & Motor skills & $\begin{array}{l}\text { Caulfield et al. [57] } \\
\text { Hamadani et al. [58] } \\
\text { Tamura et al. [55] }\end{array}$ & $\begin{array}{c}\text { Peru (2) } \\
\text { Bangladesh (4) } \\
\text { United States (1) }\end{array}$ & 3 & 985 & $-0.10(-0.38$ to 0.17$)$ & 0.49 & $72.5 \%$ \\
\hline LCPUFA & Fluid intelligence & $\begin{array}{c}\text { Brei et al. [72] } \\
\text { Dunstan et al. [46] } \\
\text { Ramakrishnan et al. [74] }\end{array}$ & $\begin{array}{l}\text { Germany (1) } \\
\text { Australia (1) } \\
\text { Mexico (2) }\end{array}$ & 3 & 999 & 0.05 ( -0.08 to 0.18$)$ & 0.45 & $10.1 \%$ \\
\hline $\mathrm{MMN}$ & Fluid intelligence & $\begin{array}{l}\text { Christian et al. [71] } \\
\text { Prado et al. [53] }\end{array}$ & $\begin{array}{c}\text { Nepal (4) } \\
\text { Indonesia (3) }\end{array}$ & 2 & 755 & 0.07 ( -0.20 to 0.33$)$ & 0.63 & $78.2 \%$ \\
\hline Zinc & Fluid intelligence & $\begin{array}{l}\text { Caulifield et al. [57] } \\
\text { Tamura et al. [55] }\end{array}$ & $\begin{array}{c}\text { Peru (2) } \\
\text { United States (1) }\end{array}$ & 2 & 539 & $-0.10(-0.25$ to 0.06$)$ & 0.23 & $0 \%$ \\
\hline MMN & Global cognition & $\begin{array}{l}\text { Joos et al. [116] } \\
\text { Li et al. [60] } \\
\text { McGrath et al. [61] } \\
\text { Waber et al. [125] } \\
\text { Chang et al. [73] } \\
\text { Christian et al. [67] }\end{array}$ & $\begin{array}{c}\text { Taiwan (2) } \\
\text { China (2) } \\
\text { Tanzania (4) } \\
\text { Colombia (2) } \\
\text { China (3) } \\
\text { Bangladesh (4) }\end{array}$ & 6 & 3126 & $0.09(-0.02$ to 0.19$)$ & 0.11 & $57.2 \%$ \\
\hline
\end{tabular}


Table 3. Cont

\begin{tabular}{|c|c|c|c|c|c|c|c|c|}
\hline Dietary Nutrient & Cognitive Outcome & Study Authors & Country (OECD) & Studies $(n)$ & Children $(n)$ & $\operatorname{SMD}(95 \% \text { CI })^{\dagger}$ & $p$-Value & $I^{2 \ddagger}$ \\
\hline LCPUFA & Global cognition & $\begin{array}{c}\text { Dunstan et al. [46] } \\
\text { Helland et al. [49] } \\
\text { Helland et al. [51] } \\
\text { Judge et al. [59] } \\
\text { Makrides et al. [52], } \\
\text { Mulder et al. [62] } \\
\text { Tofail et al. [63] } \\
\text { Van Goor et al. [56] } \\
\text { Brei et al. [72] } \\
\text { Ramakrishnan et al. [74] }\end{array}$ & $\begin{array}{c}\text { Australia (1) } \\
\text { Norway (1) } \\
\text { Norway (1) } \\
\text { United States (1) } \\
\text { Australia (1) } \\
\text { Canada (1) } \\
\text { Bangladesh (4) } \\
\text { Netherlands (1) } \\
\text { Germany (1) } \\
\text { Mexico (2) }\end{array}$ & 10 & 2632 & 0.03 ( -0.07 to 0.13$)$ & 0.55 & $21.3 \%$ \\
\hline Zinc & Crystallised intelligence & $\begin{array}{l}\text { Caulfield et al. [57] } \\
\text { Tamura et al. [55] }\end{array}$ & $\begin{array}{c}\text { Peru (2) } \\
\text { United States (1) }\end{array}$ & 2 & 539 & -0.04 ( -0.20 to 0.12$)$ & 0.61 & $0 \%$ \\
\hline LCPUFA & Crystallised intelligence & $\begin{array}{c}\text { Dunstan et al. [46] } \\
\text { Makrides et al. [52] } \\
\text { Mulder et al. [62] } \\
\text { Brie et al. [72] } \\
\text { Ramakrishnan et al. [74] }\end{array}$ & $\begin{array}{c}\text { Australia (1) } \\
\text { Australia (1) } \\
\text { Canada (1) } \\
\text { Germany (1) } \\
\text { Mexico (2) }\end{array}$ & 5 & 1941 & $0.25(-0.04$ to 0.53$)$ & 0.09 & $87.8 \%$ \\
\hline $\mathrm{MMN}$ & Crystallised intelligence & $\begin{array}{l}\text { Christian et al. [67] } \\
\text { Prado et al. [53] }\end{array}$ & $\begin{array}{l}\text { Bangladesh (4) } \\
\text { Indonesia (3) }\end{array}$ & 2 & 1207 & $0.01(-0.11$ to 0.12$)$ & 0.91 & $0 \%$ \\
\hline
\end{tabular}

CI: confidence interval; LCPUFA: long chain polyunsaturated fatty acids; MMN: multiple micronutrient; SMD: standardised mean difference; OECD: the organisation for economic

co-operation and development criteria; $1=$ high income country, $2=$ higher middle income country, $3=$ lower middle income country \& $4=$ low income country; ${ }^{\dagger}$ the main measure of effect was SMD (also known as Cohens d). The SMD was determined by taking the difference between the mean of the intervention group compared to the control group, and dividing the pooled standard deviation for the outcome across the whole trial. A random effects model using the method DerSimonian \& Laird [43] was applied to the data; $¥$ the $I^{2}$ statistic is the percentage of observed total variation across studies that is due to heterogeneity rather than chance. It is calculated using the following formula: $I^{2}=100 \% \times(Q-d f) / Q$, where $Q$ is Cochran's heterogeneity and $d f$ is the degrees of freedom [44]. 


\subsection{Outcome 2: Memory}

Four RCTs $[45,55,65,74]$ measured the effect of a nutritional intervention on memory composite score. There was no significant effect of iron supplementation ( $n=1$ trial) [55], zinc supplementation ( $n=1$ trial) [65], choline supplementation ( $n=1$ trial) [45] or LCPUFA supplementation $(n=1$ trial) [74] on memory composite scores.

\subsection{Outcome 3: Motor Skills}

Eighteen RCTs $[46,48,52,53,55-58,60-64,67,71-74]$ measured the effect of a nutritional intervention on the motor skills score. Multiple outcomes were combined to create a composite score in Brei et al. [72] (2 outcomes), Dunstan et al. [46] (2 outcomes), Mulder et al. [62] (2 outcomes), and Christian et al. [71] (2 outcomes). Li et al. [60] Chang et al. [73], and Christian et al. [71] used two interventions and one control group, the sample populations were not independent, therefore the effect sizes were combined. McGrath et al. [61] also used multiple independent intervention and control groups, therefore the effect sizes were kept separate. There was no significant effect of multiple micronutrient interventions ( $n=6$ trials) $[53,60,61,67,71,73]$, LCPUFA supplementation ( $n=7$ trials) $[46,52,56,62,63,72,74]$, zinc supplementation ( $n=3$ trials) $[55,57,58]$ or food-based intervention ( $n=1$ trial) [64] on motor skills composite scores (Table 3 and Figure 3). Hanieh et al. [48] investigated the effect of nutrient supplementation of varying dosage on cognition, no significant effect on infant motor skills composite score was reported. The heterogeneity among the zinc intervention trials was significant $(p=0.03)$. From the statistical tests of Egger $(p=0.54)$, there was no evidence of publication bias (Supplementary Figure S4). The heterogeneity among the zinc intervention trials was significant $(p=0.03)$. From the sensitivity analysis, the country-income of the studies did not significantly $(p>0.05)$ affect child motor skills (Supplementary Figure S5).

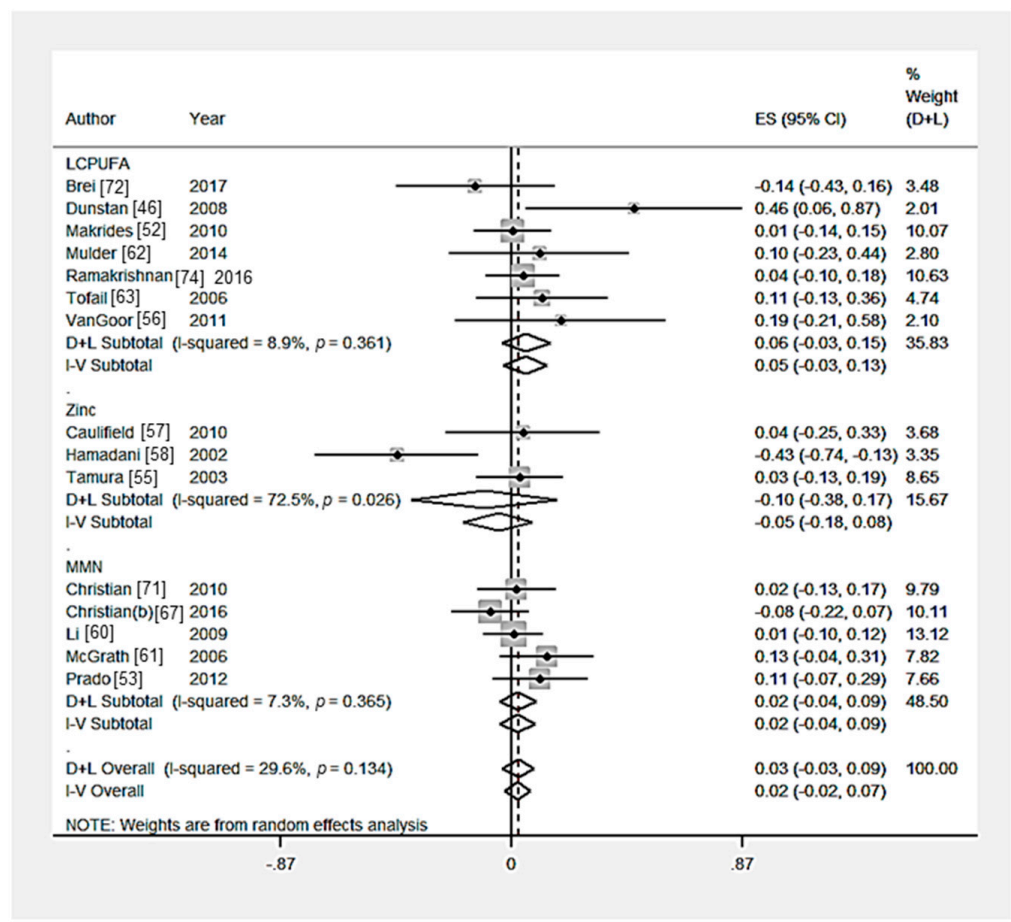

Figure 3. Forest plot for child motor skills outcomes. The overall effect size was estimated by standardised mean difference (SMD). LCPUFA, long chain polyunsaturated fatty acids; MMN, multiple micronutrient. D+L, random-effects estimate (Der Simonian and Laird method) and I-V, fixed-effects estimate (inverse variance method). 


\subsection{Outcome 4: Fluid Intelligence}

Eight RCTs $[46,53,55,65,71,72,74]$ measured the effect of a nutritional intervention on the fluid intelligence composite score. Multiple outcomes were combined to create a composite score in Brei et al. [72] (2 outcomes), and Ramakrishnan et al. [74] (2 outcomes).There was no significant effect of LCPUFA supplementation ( $n=3$ trials) [46,72,74], multiple micronutrient supplementation ( $n=2$ trials) [53,67], zinc supplementation ( $n=2$ trials) [55,57] or iron supplementation ( $n=1$ trial) [65] on fluid intelligence composite scores (Table 3 and Figure 4). From the statistical tests of Egger $(p=0.39)$, there was no evidence of publication bias (Supplementary Figure S6). From the sensitivity analysis, the country-income of the studies did not significantly $(p>0.05)$ affect child fluid intelligence (Supplementary Figure S7).

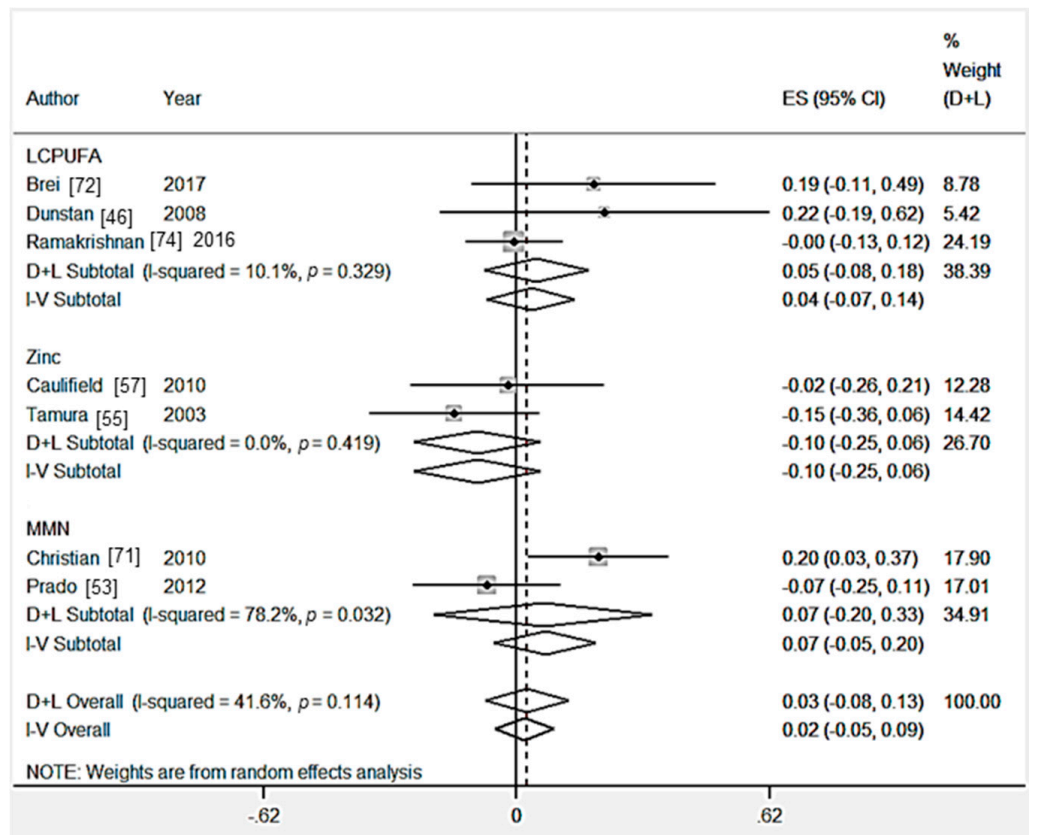

Figure 4. Forest plot for child fluid intelligence outcomes. The overall effect size was estimated by standardised mean difference (SMD). LCPUFA, long chain polyunsaturated fatty acids; MMN, multiple micronutrient. D+L, random-effects estimate (Der Simonian and Laird method) and I-V, fixed-effects estimate (inverse variance method).

\subsection{Outcome 5: Global Cognition}

Twenty RCTs $[46,48,49,51,52,56,57,59-63,65,67,69,72-74,116,125]$ measured the effect of a nutritional intervention on global cognition. The effect size of multiple time points were combined in Chang et al. [73], Helland et al. [49], Helland et al. [51] and Helland et al. [50]. Li et al. [60] Van Goor et al. [56] Chang et al. [73] and Waber et al. [125] used multiple interventions and one control group, the sample populations were not independent, therefore the effect sizes were combined. McGrath et al. [61] also used multiple independent intervention and control groups, therefore the effect sizes were kept separate. There was no significant effect of multiple micronutrient interventions ( $n=6$ trials) $[60,61,67,73,116,125]$ LCPUFA interventions ( $n=10$ trials) $[46,49,51,52,56,59,62,63,72,74]$ iron supplementation ( $n=1$ trial) [65] or vitamin A supplementation ( $n=1$ trial) [69] on global cognition composite scores (Table 3 and Figure 5). Hanieh et al. [48] investigated the effect of nutrient supplementation of varying dosage on cognition, iron and folic acid supplements given twice weekly was shown to significantly effect global cognition composite scores in infants at six months. The heterogeneity among the multiple micronutrient intervention trials was significant $(p=0.04)$. From the statistical tests of Egger $(p=0.82)$, there was no evidence of publication bias 
(Supplementary Figure S8). From the sensitivity analysis, the country-income of the studies did not significantly $(p>0.05)$ affect child global cognition outcomes (Supplementary Figure S9).

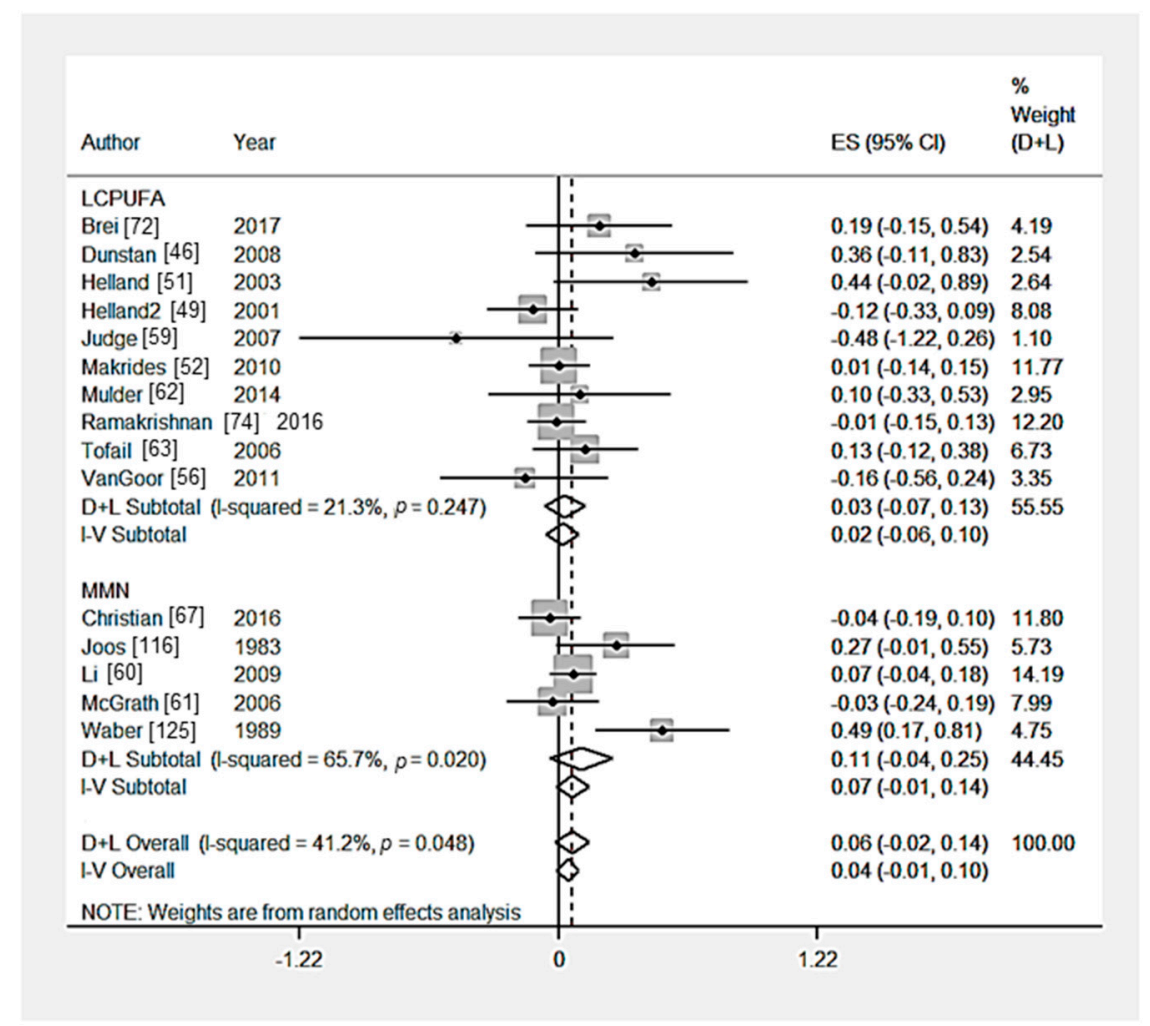

Figure 5. Forest plot for child global cognition outcomes. The overall effect size was estimated by standardised mean difference (SMD). LCPUFA, long chain polyunsaturated fatty acids; MMN, multiple micronutrient. D+L, random-effects estimate (Der Simonian and Laird method) and I-V, fixed-effects estimate (inverse variance method).

\subsection{Outcome 6: Crystallised Intelligence}

Eleven RCTs $[46,48,52,53,55,57,62,65,67,72,74]$ measured the effect of a nutritional intervention on crystallised intelligence composite score. Multiple outcomes were combined to create a composite score in Brei et al. [72] (2 outcomes), Caulfield et al. [57] (3 outcomes), Dunstan et al. [46] (4 outcomes) and Mulder et al. [62] (7 outcomes). There was no significant effect of LCPUFA supplementation ( $n=5$ trials) [46,52,62,72,74], multiple micronutrient supplementation [53,67] ( $n=2$ trials), zinc supplementation ( $n=2$ trials) [55,57] or iron supplementation ( $n=1$ trial) [65] on crystallised intelligence composite scores (Table 3 and Figure 6). Hanieh et al. [48] investigated the effect of nutrient supplementation of varying dosage on cognition, no significant effect on child crystallised intelligence composite score was reported. The heterogeneity among the LCPUFA intervention trials was significant $(p=0.00)$. From the statistical tests of Egger $(p=0.05)$, there was no evidence of publication bias, however there is asymmetry in the funnel plot (Figure 7). From the sensitivity analysis, the country-income of the studies did not significantly $(p>0.05)$ affect child crystallised intelligence (Supplementary Figure S10). 


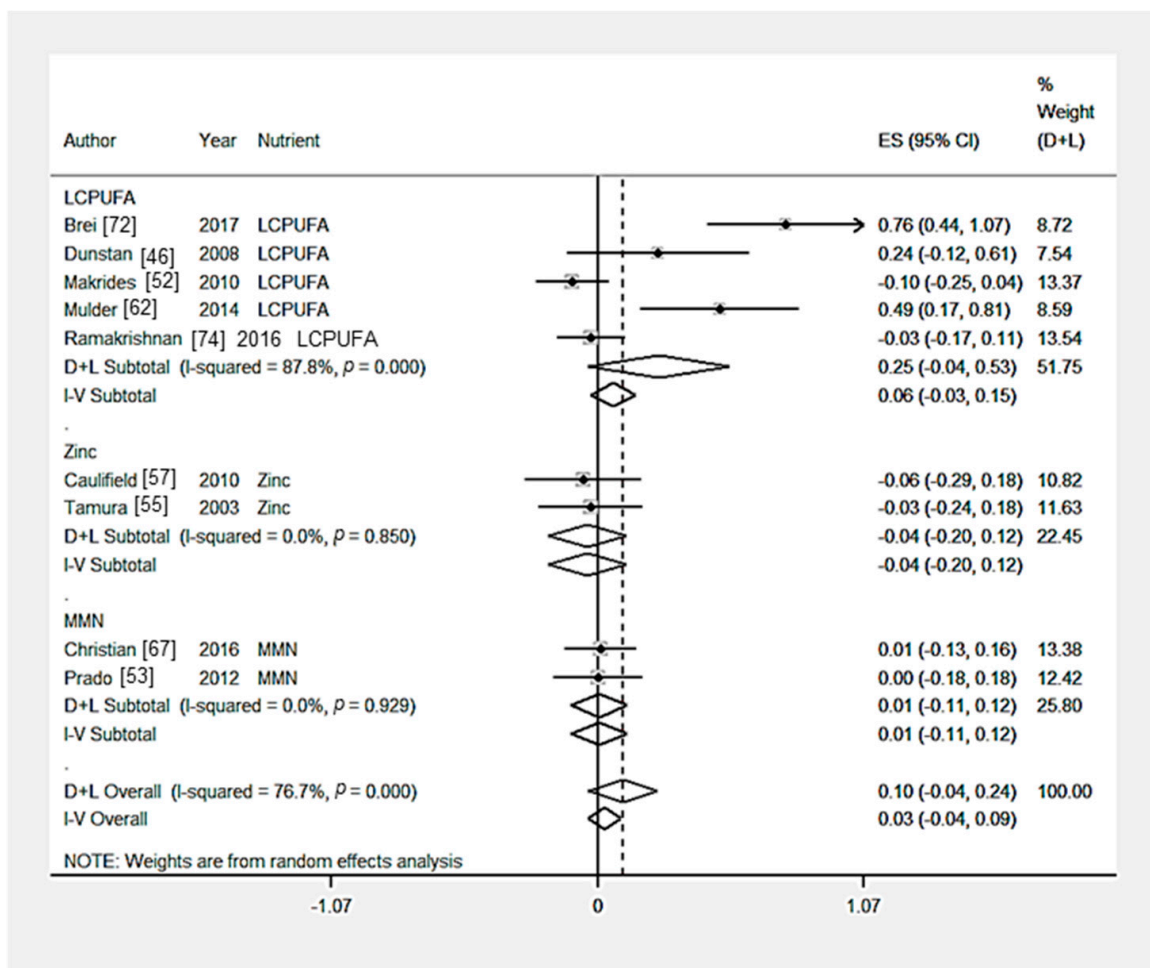

Figure 6. Forest plot for child crystallised intelligence outcomes. The overall effect size was estimated by standardised mean difference (SMD). LCPUFA, long chain polyunsaturated fatty acids; MMN, multiple micronutrient. D+L, random-effects estimate (Der Simonian and Laird method) and I-V, fixed-effects estimate (inverse variance method).

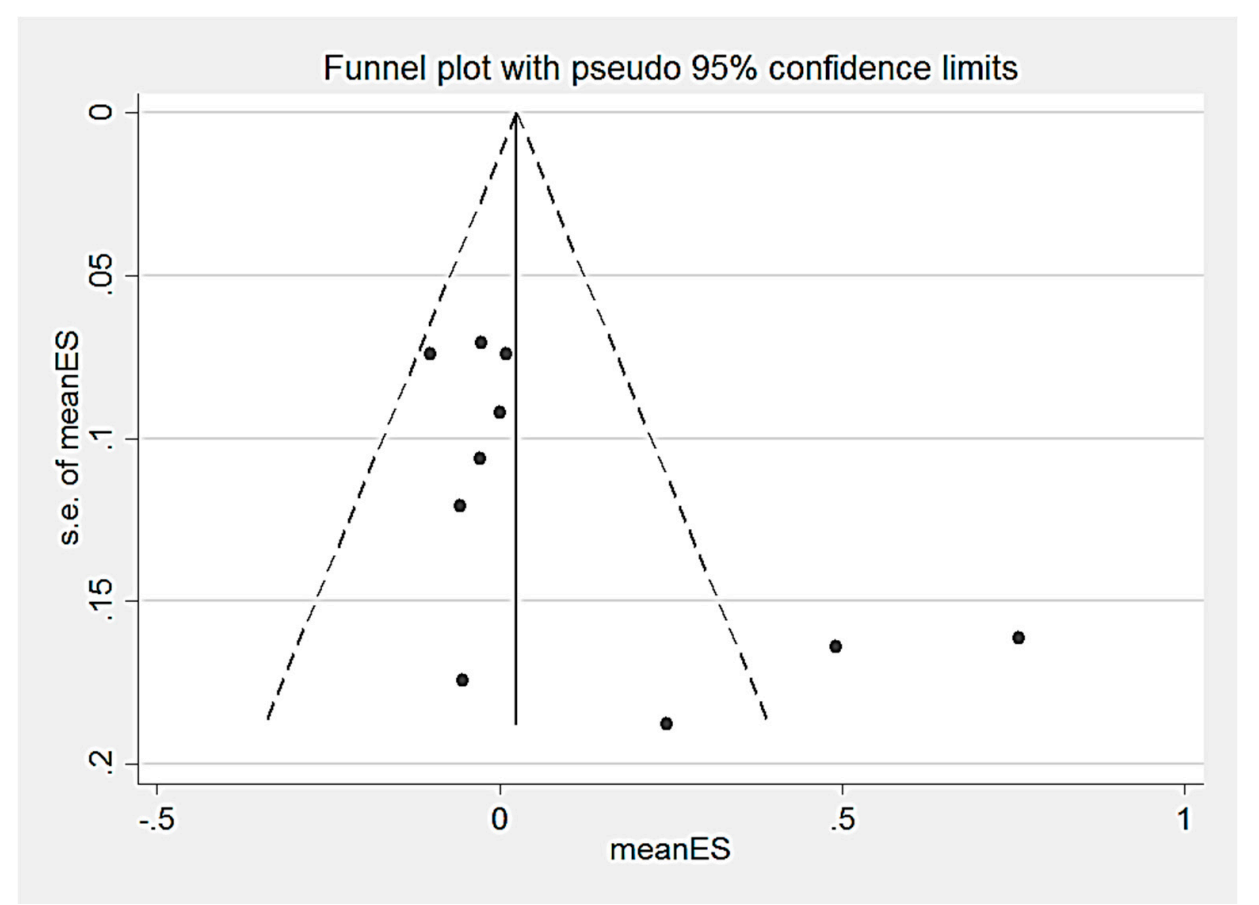

Figure 7. Funnel plot for child crystallized intelligence outcomes with $95 \%$ confidence limits. meanSE, mean standard error. 


\subsection{Outcome 7: Visual Processing}

Three RCTs $[45,53,55]$ measured the effect of a nutritional intervention on visual processing. There was no significant effect of choline supplementation ( $n=1$ trial) [45], multiple micronutrient supplementation ( $n=1$ trial) [53] or zinc supplementation ( $n=1$ trial) [55] on visual processing composite scores.

\subsection{Outcome 8: Attention}

Four RCTs $[47,66,74,76]$ measured the effect of a nutritional intervention on attention. There was no significant effect of multiple or LCPUFA supplementation ( $n=2$ trials) $[47,74]$ on attention. Vuori et al. [76] reported that multiple micronutrient supplementation during pregnancy did positively affect attention in infants at 15 days postnatally.

\subsection{Outcome 9: Problem Solving}

Two RCTs [59,64] measured the effect of a nutritional intervention on the problem solving composite score. There was no significant effect of multiple micronutrient intervention ( $n=1$ trial) [64] or LCPUFA intervention ( $n=1$ trial) [59] on problem composite scores.

\section{Discussion}

This systematic review and meta-analysis indicates that nutritional interventions during pregnancy do not significantly impact on child cognitive outcomes. There is some evidence to suggest that LCPUFA supplementation may marginally improve child crystallised intelligence, however the effect was not statistically significant and study heterogeneity among the trials was significant. Although a wide scope of literature was assessed and a rigorous selection methodology was applied, the data included in this systematic review were affected by significant methodological limitations. From the included studies, $79 \%$ were exclusively nutrient supplementation trials, the effect of food-based interventions or nutrition counselling alone during pregnancy on child cognition was not well explored. Many studies were small, and attrition rates were high due to the length of follow-up required. Child cognition was a secondary outcome of interest in many studies, even with meta-analysis our findings are likely to be underpowered. This systematic was unable to perform a meta-analysis in specific age ranges due to the lack of data available for children older than 35 months, especially in low and middle income countries. Therefore, it is important that future trials include a longer child follow-up, to explore any possible delayed effects of maternal nutritional interventions on child cognition.

\subsection{Quality of Included Studies}

Many included studies were considered to be of high quality. While, the Quality Checklist Criteria [36] is designed to assess the methodological quality and reporting within an article, it does not assess the appropriateness of the methodology in addressing the research question. This is a concern considering that many of included RCTs are likely to be at risk of type 2 error, due to the use of secondary data analysis and not powered to detect differences in cognitive outcome as a primary outcome variable. In addition, many post-hoc studies excluded participants after randomisation and in some cases multiple variable adjustments were required to address the significant characteristic differences between the groups, suggesting the assumptions of randomisation had been breached. Most post-hoc studies did not perform intention-to-treat analysis to evaluate the randomisation of the study groups. This approach is likely to introduce bias and systematic error that will reduce the power of the study. Therefore, it is important that future evidence is derived from adequately powered primary studies to reduce the risk of a type 2 error and systematic bias in post-hoc studies and determine whether there are any effects of nutritional interventions during pregnancy on child cognition. 


\subsection{Measuring Child Cognition}

There are many methodological challenges when measuring child cognition using psychological assessment tests. The cognitive performance of infants and children will be affected by their mood, motivation, amount of sleep and personal effort [138]. Environmental factors, including who conducts the cognition tests, who else is present during testing and where the tests are held can also influence a child's performance ability [138]. In addition, most cognition tests were devised in developed countries, using stimuli relevant to their culture, therefore this stimuli is likely to be unfamiliar or unacceptable to children from developing countries.

Many studies in this review used global development assessments (e.g., Bayley's Scales of Infant Development) to determine the children's overall cognitive ability. However, broad measures of cognitive function may only detect highly significant nutritional effects, especially if the study is underpowered [138]. Specific cognitive skills (e.g., attention, memory) that contribute to overall function are regulated by distinct neural mechanisms, that may be differentially affected by nutritional manipulations [139]. Therefore, Cognitive Assessment Tests that analyse specific skills, may be more sensitive to detect subtle effects of nutritional interventions on cognition. For this reason, future trials may be improved by the use of assessment tests that measure specific cognitive skills, as well as global measures of cognition. The choice of specific cognition test should be based on the hypothesised neural mechanisms that are expected to be altered by nutrients.

To determine cognitive function later in childhood and adolescence, future studies should consider linking and analysing school performance data. Pilot data should be used to determine the appropriateness of the sample size and ensure that the study is adequately powered. This approach also has the advantage of the outcome assessors being blinded to the randomisation.

\subsection{Nutrition Intervention Design}

Due to methodological and ethical challenges, most trials analysed the effects of nutrient supplementation during pregnancy on child cognition rather than using food-based interventions. This may reflect the perception that obtaining adherence to food-based interventions is a challenge and therefore nutrient supplementation is a more feasible strategy/study design. However, performing a meta-analysis for the supplementation trials proved to be difficult because many studies used a supplemented control group rather than an unsupplemented placebo group. Consequently, only the effect of the additional nutrient supplemented in the intervention group compared to the control group could be accessed. In Australia, evidence-based guidelines recommend the use of folic acid and iodine supplementation during pregnancy, therefore it is unethical to use an unsupplemented control group within this population. Li et al. [60] and Caulfield et al. [57] attempted to address this dilemma by providing the same dosage of folic acid across all study groups while women in the intervention groups received a multiple micronutrient supplement (including folic acid) or folic acid with iron. In addition, $88 \%$ of the studies conducted in high-income countries provided a single nutrient supplement to women during pregnancy. Since the effect of a single nutrient supplement on child cognition is likely to be modest, especially in a population that is well-nourished, this is likely to explain why a significant summary effect was not found. While, $88 \%$ of middle and low-income country studies, provided multiple micronutrient supplementation in a population that is likely to be undernourished, but no significant effect on child cognition was found. However, since child cognition assessment test were validated in a western population, this may have resulted in summary effect that was underestimated.

By convention, many randomised control trials analyse the effect of nutrition on cognition by distilling complex dietary patterns into a single agent using a nutrient supplement. A single nutrient supplement is However, the bioavailability and efficacy of nutrient supplements can differ compared to nutrients contained in food [140]. Nutrient bioavailability is affected by the specific nutrient, absorptive capacity of intestinal mucosa, physiological states (e.g., pregnancy) and nutrient-nutrient interactions [140]. For example, the bioavailability of inorganic iron is enhanced by 
an interaction with vitamin $\mathrm{C}$ and increasing the bioavailability of iron can decrease magnesium and calcium absorption [140]. Huang et al. [141] reported that the bioavailability of natural vitamin was approximately 50 percent greater than synthetic vitamin E ( $\alpha$-tocopherol) in adults. In addition, synthetic vitamin E supplementation was also associated with a significant reduction in the bioavailability of other forms of vitamin E [141]. This trial highlights the limitations of analysing the effects of nutrition via a single agent without considering the biochemical pathways in which nutrients interact that is likely to be important for cognitive development [142-144]. Given the limited evidence available, there is a clear need for trials that are designed from a sound biologically plausible basis.

\subsection{Public Health Implications}

Based on the meta-analyses presented, there is a lack of robust evidence on which to base recommendations for maternal diet or for the use of nutrient supplements as a strategy to enhance cognition in children. Given that the synergistic effect of micronutrients in metabolic pathways is important for enhancing cognitive development of children [145], it is important that future trials focus on food-based interventions that aim to improve the overall diet quality of pregnant women. In developing countries, pregnant women are more likely to experience protein-energy malnutrition, as well as multiple micronutrient deficiencies in comparison to high-income countries [146]. Therefore, further evidence is required to determine whether protein and energy as well as micronutrients interventions are effective for improving child cognition in the long-term in developing countries.

\section{Conclusions}

In conclusion, this systematic review suggests that the infant and child cognitive outcomes were not significantly improved by nutritional interventions (dietary counselling, education, nutrition supplementation, fortified foods and/or whole foods). However, the possible association between LCPUFA supplementation and child crystallised intelligence warrants further investigation in high quality RCTs powered to detect difference in cognitive variables as the primary outcome. Further evidence from primary research is required to test whether maternal nutritional interventions during pregnancy, in particular food-based interventions, are beneficial for improving child cognition. This review emphasises the need for well-designed pilot studies to inform adequately powered RCTs and data linkage studies. This evidence is important because gains in child cognition are likely to have extended reach to other areas of public health and carry economic benefit.

Supplementary Materials: The following are available online at www.mdpi.com/2072-6643/9/11/1265/s1, Table S1: Search strategy used for systematic review; Table S2: Computing a combined effect across outcomes; Table S3: Summary of quality assessment; Table S4: Summary of the nutritional interventions for included studies; Table S5: Cognitive Assessment Tests; Figure S1: Review protocol; Figure S2: Funnel plot: behavior; Figure S3: Sensitivity analysis: behavior; Figure S4: Funnel plot: motor skills; Figure S5: Sensitivity analysis: motor skills; Figure S6: Funnel plot: fluid intelligence; Figure S7: Sensitivity analysis: fluid intelligence; Figure S8: Funnel plot: global cognition; Figure S9: Sensitivity analysis: global cognition; Figure S10: Sensitivity analysis: crystallised intelligence.

Acknowledgments: We wish to thank Debbie Booth for her assistance in developing the literature search strategy. This work was supported by a University of Newcastle PhD Scholarship to R.M.T.

Author Contributions: R.M.T., A.J.H., A.B., C.E.C., R.S. were involved in the research design. R.M.T. and S.F. conducted the research including literature search, quality assessment and data extraction. A.J.H. assisted with publication eligibility and assessing methodological quality. R.M.T., A.B. and T.-J.E. performed the statistical analysis. R.M.T. drafted the paper and was primary responsible for the final content. All authors provided constructive feedback and approved the final manuscript.

Conflicts of Interest: The authors declare no conflict of interest. 


\section{Abbreviations}

$\begin{array}{ll}\text { CHC } & \text { Cattell-Horn-Carroll } \\ \text { CI } & \text { confidence interval } \\ \text { DHA } & \text { docosahexaenoic acid } \\ \text { HIV-1 } & \text { human immunodeficiency virus type 1 } \\ \text { LCPUFA } & \text { long chain polyunsaturated fatty acids } \\ \text { OECD } & \text { Organisation for Economic Co-operation and Development } \\ \text { PRISMA } & \text { Preferred Reporting Items for Systematic Review Meta-Analyses } \\ \text { PRISMA } & \text { randomised controlled trials } \\ \text { SMD } & \text { standardised mean difference }\end{array}$

\section{References}

1. Couperus, J.W.; Nelson, C.A. Early brain development and plasticity. In The Blackwell Handbook of Early Childhood Development; McCartney, K., Phillips, D., Eds.; Blackwell Publishing Ltd.: Malden, MA, USA, 2006; pp. 85-105.

2. Hibbard, E.D.; Smithells, R.W. Folic acid metabolism and human embryopathy. Lancet 1965, $285,1254$. [CrossRef]

3. Wald, N.; Sneddon, J. Prevention of neural tube defects: Results of the Medical Research Council Vitamin Study. Lancet 1991, 338, 131-137. [CrossRef]

4. Smithells, R.W.; Sheppard, S.; Schorah, C.J. Vitamin deficiencies and neural tube defects. Arch. Dis. Child. 1976, 51, 944-950. [CrossRef] [PubMed]

5. Smithells, R.W.; Sheppard, S.; Schorah, C.J.; Seller, M.J.; Nevin, N.C.; Harris, R.; Read, A.P.; Fielding, D.W. Apparent prevention of neural tube defects by periconceptional vitamin supplementation. Arch. Dis. Child. 1981, 56, 911-918. [CrossRef] [PubMed]

6. Czeizel, A.E.; Dudas, I. Prevention of the first occurrence of neural-tube defects by periconceptional vitamins supplementation. N. Engl. J. Med. 1992, 327, 1832-1835. [CrossRef] [PubMed]

7. Thompson, R.A.; Nelson, C.A. Developmental science and the media. Early brain development. Am. Psychol. 2001, 56, 5-15. [CrossRef] [PubMed]

8. Bernal, J.; Nunez, J. Thyroid hormones and brain development. Eur. J. Endocrinol. 1995, 133, $390-398$. [CrossRef] [PubMed]

9. Rao, R.; Tkac, I.; Townsend, E.L.; Ennis, K.; Gruetter, R.; Georgieff, M.K. Perinatal iron deficiency predisposes the developing rat hippocampus to greater injury from mild to moderate hypoxia-ischemia. J. Cereb. Blood Flow Metab. 2007, 27, 729-740. [CrossRef] [PubMed]

10. Ward, K.L.; Tkac, I.; Jing, Y.; Felt, B.; Beard, J.; Connor, J.; Schallert, T.; Georgieff, M.K.; Rao, R. Gestational and lactational iron deficiency alters the developing striatal metabolome and associated behaviors in young rats. J. Nutr. 2007, 137, 1043-1049. [PubMed]

11. Wiesinger, J.A.; Buwen, J.P.; Cifelli, C.J.; Unger, E.L.; Jones, B.C.; Beard, J.L. Down-regulation of dopamine transporter by iron chelation in vitro is mediated by altered trafficking, not synthesis. J. Neurochem. 2007, 100, 167-179. [CrossRef] [PubMed]

12. Lozoff, B.; De Andraca, I.; Castillo, M.; Smith, J.B.; Walter, T.; Pino, P. Behavioral and Developmental Effects of Preventing Iron-Deficiency Anemia in Healthy Full-Term Infants. Pediatrics 2003, 112, 846-854. [PubMed]

13. Fox, S.E.; Levitt, P.; Nelson, C.A. How the Timing and Quality of Early Experiences Influence the Development of Brain Architecture. Child. Dev. 2010, 81, 28-40. [CrossRef] [PubMed]

14. Cusick, S.E.; Georgieff, M.K. The Role of Nutrition in Brain Development: The Golden Opportunity of the "First 1000 Days". J. Pediatr. 2016, 175, 16-21. [CrossRef] [PubMed]

15. Sampaio, R.C.; Truwit, C.L. Myelination in the developing human brain. In Handbook of Developmental Cognitive Neuroscience; Nelson, C.A., Luciana, M., Eds.; MIT Press: Cambridge, MA, USA, 2001; pp. $35-44$.

16. Thatcher, R.W. Maturation of the human frontal lobes: Physiological evidence for staging. Dev. Neuropsychol. 1991, 7, 397-419. [CrossRef]

17. Bryan, J.; Osendarp, S.; Hughes, D.; Calvaresi, E.; Baghurst, K.; Van Klinken, J.W. Nutrients for cognitive development in school-aged children. Nutr. Rev. 2004, 62, 295-306. [CrossRef] [PubMed] 
18. Anjos, T.; Altmae, S.; Emmett, P.; Tiemeier, H.; Closa-Monasterolo, R.; Luque, V.; Wiseman, S.; Pérez-García, M.; Lattka, E.; Demmelmair, H.; et al. Nutrition and neurodevelopment in children: Focus on NUTRIMENTHE project. Eur. J. Nutr. 2013, 52, 1825-1842. [CrossRef] [PubMed]

19. Prado, E.L.; Dewey, K.G. Nutrition and brain development in early life. Nutr. Rev. 2014, 72, $267-284$. [CrossRef] [PubMed]

20. Florence, M.; Asbridge, M.; Veugelers, P.J. Diet quality and academic performance. J. School Health 2008, 78, 209-215. [CrossRef] [PubMed]

21. Farrington, D.P. Early predictors of adolescent aggression and adult violence. Violence Vict. 1989, 4, 79-100. [PubMed]

22. Kaslow, F.W.; Lipsitt, P.D.; Buka, S.L.; Lipsitt, L.P. Family law issues in family therapy practice: Early intelligence scores and subsequent delinquency: A Prospective study. Am. J. Fam. Ther. 1990, 18, 197-208. [CrossRef]

23. Hinshaw, S.P. Externalizing behavior problems and academic underachievement in childhood and adolescence: Causal relationships and underlying mechanisms. Psychol. Bull. 1992, 111, 127-155. [CrossRef] [PubMed]

24. Fergusson, D.M.; Horwood, L.J.; Ridder, E.M. Show me the child at seven II: Childhood intelligence and later outcomes in adolescence and young adulthood. J. Child Psychol. Psychiatry Allied Discip. 2005, 46, 850-858. [CrossRef] [PubMed]

25. Stattin, H.; Klackenberg-Larsson, I. Early language and intelligence development and their relationship to future criminal behavior. J. Abnorm. Psychol. 1993, 102, 369-378. [CrossRef] [PubMed]

26. Frisell, T.; Pawitan, Y.; Långström, N. Is the Association between General Cognitive Ability and Violent Crime Caused by Family-Level Confounders? PLoS ONE 2012, 7, e41783. [CrossRef] [PubMed]

27. Wildeman, C.; Muller, C. Mass Imprisonment and Inequality in Health and Family Life. Annu. Rev. Law Soc. Sci. 2012, 8, 11-30. [CrossRef]

28. Larson, L.M.; Yousafzai, A.K. A meta-analysis of nutrition interventions on mental development of children under-two in low- and middle-income countries. Mater. Child Nutr. 2017, 13. [CrossRef] [PubMed]

29. Liberati, A.; Altman, D.; Tetzlaff, J.; Mulrow, C.; Gøtzsche, P.C.; Ioannidis, J.P.A.; Clarke, M.; Devereaux, P.J.; Kleijnen, J.; Moher, D. The PRISMA Statement for Reporting Systematic Reviews and Meta-Analyses of Studies That Evaluate Health Care Interventions: Explanation and Elaboration. PLoS Med. 2009, 339. [CrossRef]

30. Gresham, E.; Bisquera, A.; Byles, J.E.; Hure, A.J. Effects of dietary interventions on pregnancy outcomes: A systematic review and meta-analysis. Mater. Child Nutr. 2014, 12, 5-23. [CrossRef] [PubMed]

31. McCall, R.B. Childhood IQ as Predictors of Adult Educational and Occupational Status. Science 1977, 197, 482-483. [CrossRef] [PubMed]

32. Carroll, J. Human Cognitive Abilities: A Survey of Factor-Analytic Studies; Cambridge University Press: New York, NY, USA, 1993.

33. Fernandes, M.; Stein, A.; Newton, C.R.; Cheikh-Ismail, L.; Kihara, M.; Wulff, K.; de León Quintana, E.; Aranzeta, L.; Soria-Frisch, A.; Acedo, J.; et al. The INTERGROWTH-21st Project Neurodevelopment Package: A Novel Method for the Multi-Dimensional Assessment of Neurodevelopment in Pre-School Age Children. PLoS ONE 2014, 9, e113360. [CrossRef] [PubMed]

34. Bayley, N. Bayley Scales of Infant Development, 3rd ed.; Harcourt Assessment: San Antorio, TX, USA, 2005.

35. Eilander, A.; Gera, T.; Sachdev, H.S.; Transler, C.; van der Knaap, H.C.; Kok, F.J.; Osendarp, S.J. Multiple micronutrient supplementation for improving cognitive performance in children: Systematic review of randomized controlled trials. Am. J. Clin. Nutr. 2010, 91, 115-130. [CrossRef] [PubMed]

36. Academy of Nutrition and Dietetics. Evidence Analysis Manual: Steps in the Academy Evidence Analysis Process; ADA Research and Strategic Business Development: Chicago, IL, USA, 2012.

37. The World Bank Group. Country and Lending Groups: High Income OCED Members 2015. Available online: http://data.worldbank.org/about/country-and-lending-groups\#OECD_members (accessed on 23 January 2015).

38. Gera, T.; Sachdev, H.P.S.; Nestel, P. Effect of combining multiple micronutrients with iron supplementation on $\mathrm{Hb}$ response in children: Systematic review of randomized controlled trials. Public Health Nutr. 2009, 12, 756-773. [CrossRef] [PubMed] 
39. Roberts, J.L.; Stein, A.D. The Impact of Nutritional Interventions beyond the First 2 Years of Life on Linear Growth: A Systematic Review and Meta-Analysis. Adv. Nutr. 2017, 8, 323-336. [CrossRef] [PubMed]

40. Murphy, S.P.; White, K.K.; Park, S.Y.; Sharma, S. Multivitamin-multimineral supplements' effect on total nutrient intake. Am. J. Clin. Nutr. 2007, 85, 280S-284S. [PubMed]

41. Sanchez-Meca, J.; Marin-Martinez, F.; Chacon-Moscoso, S. Effect-size indices for dichotomized outcomes in meta-analysis. Psychol. Methods 2003, 8, 448-467. [CrossRef] [PubMed]

42. Borenstein, M.; Hedges, L.V.; Higgins, J.P.T.; Rothstein, H.R. Effect Sizes Based on Correlations. In Introduction to Meta-Analysis; John Wiley \& Sons, Ltd.: Hoboken, NJ, USA, 2009; pp. 41-43.

43. DerSimonian, R.; Laird, N. Meta-analysis in clinical trials. Control. Clin. Trials 1986, 7, 177-188. [CrossRef]

44. Higgins, J.P.T.; Thompson, S.G.; Deeks, J.J.; Altman, D.G. Measuring inconsistency in meta-analyses. BMJ 2003, 327, 557-560. [CrossRef] [PubMed]

45. Cheatham, C.; Davis Goldman, B.; Fischer, L.; da Costa, K.A.; Reznick, J.S.; Zeisel, S.H. Phosphatidylcholine supplementation in pregnant women consuming moderate-choline diets does not enhance infant cognitive function: A randomized, double-blind, placebo-controlled trial. Am. J. Clin. Nutr. 2012, 96, 1465-1472. [CrossRef] [PubMed]

46. Dunstan, J.A.; Simmer, K.; Dixon, G.; Prescott, S.L. Cognitive assessment of children at age 2(1/2) years after maternal fish oil supplementation in pregnancy: A randomised controlled trial. Arch. Dis. Child. Fetal Neonatal Ed. 2008, 93, F45-F50. [CrossRef] [PubMed]

47. Gould, J.; Makrides, M.; Colombo, J.; Smithers, L. Randomized controlled trial of maternal omega-3 long-chain PUFA supplementation during pregnancy and early childhood development of attention, working memory, and inhibitory control. Am. J. Clin. Nutr. 2014, 99, 851-859. [CrossRef] [PubMed]

48. Hanieh, S.; Ha, T.T.; Simpson, J.A.; Casey, G.J.; Khuong, N.C.; Thoang, D.D.; Thung, T.T.; Pasricha, R.S.; Tran, T.D.; Tuan, T.; et al. The effect of intermittent antenatal iron supplementation on maternal and infant outcomes in rural Viet Nam: A cluster randomised trial. PLoS Med. 2013, 10, e1001470. [CrossRef] [PubMed]

49. Helland, I.; Saugstad, O.; Smith, L.; Saarem, K.; Solvoll, K.; Ganes, T.; Drevon, C. A. Similar effects on infants of $n-3$ and $n-6$ fatty acids supplementation to pregnant and lactating women. Pediatrics 2001, 108, E82. [CrossRef] [PubMed]

50. Helland, I.; Smith, L.; Blomen, B.; Saarem, K.; Saugstad, O.; Drevon, C. Effect of supplementing pregnant and lactating mothers with $n-3$ very-long-chain fatty acids on children's IQ and body mass index at 7 years of age. Pediatrics 2008, 122, e472-e479. [CrossRef] [PubMed]

51. Helland, I.; Smith, L.; Saarem, K.; Saugstad, O.; Drevon, C. Maternal supplementation with very-long-chain n-3 fatty acids during pregnancy and lactation augments children's IQ at 4 years of age. Pediatrics 2003, 111, e39-e44. [CrossRef] [PubMed]

52. Makrides, M.; Gibson, R.A.; McPhee, A.J.; Yelland, L.; Quinlivan, J.; Ryan, P. Effect of DHA supplementation during pregnancy on maternal depression and neurodevelopment of young children: A randomized controlled trial. JAMA 2010, 304, 1675-1683. [CrossRef] [PubMed]

53. Prado, E.L.; Ullman, M.T.; Muadz, H.; Alcock, K.J.; Shankar, A.H. The effect of maternal multiple micronutrient supplementation on cognition and mood during pregnancy and postpartum in indonesia: A randomized trial. PLoS ONE 2012, 7, e32519. [CrossRef] [PubMed]

54. Santiago, P.; Velasco, I.; Muela, J.A.; Sanchez, B.; Martinez, J.; Rodriguez, A.; Berrio, M.; Gutierrez Repiso, C.; Carreira, M.; Monreno, A.; et al. Infant neurocognitive development is independent of the use of iodised salt or iodine supplements given during pregnancy. Br. J. Nutr. 2013, 110, 831-839. [CrossRef] [PubMed]

55. Tamura, T.; Goldenberg, R.L.; Ramey, S.L.; Nelson, K.G.; Chapman, V.R. Effect of zinc supplementation of pregnant women on the mental and psychomotor development of their children at $5 \mathrm{y}$ of age. Am. J. Clin. Nutr. 2003, 77, 1512-1516. [PubMed]

56. Van Goor, S.A.; Dijck-Brouwer, D.A.J.; Erwich, J.; Schaafsma, A.; Hadders-Algra, M. The influence of supplemental docosahexaenoic and arachidonic acids during pregnancy and lactation on neurodevelopment at eighteen months. Prostaglandins Leukot. Essent. Fat. Acids 2011, 84, 139-146. [CrossRef] [PubMed]

57. Caulfield, L.; Putnick, D.; Zavaleta, N.; Lazarte, F.; Albornoz, C.; Dipietro, J.; Chen, P.; Bornstein, M. Maternal gestational zinc supplementation does not influence multiple aspects of child development at 54 mo of age in Peru. Am. J. Clin. Nutr. 2010, 92, 130-136. [CrossRef] [PubMed] 
58. Hamadani, J.D.; Fuchs, G.J.; Osendarp, S.J.; Huda, S.N.; Grantham-McGregor, S.M. Zinc supplementation during pregnancy and effects on mental development and behaviour of infants: A follow-up study. Lancet 2002, 360, 290-294. [CrossRef]

59. Judge, M.; Harel, O.; Lammi-Keefe, C. Maternal consumption of a docosahexaenoic acid-containing functional food during pregnancy: Benefit for infant performance on problem-solving but not on recognition memory tasks at age 9 mo. Am. J. Clin. Nutr. 2007, 85, 1572-1577. [PubMed]

60. Li, Q.; Yan, H.; Zeng, L.; Cheng, Y.; Liang, W.; Dang, S.; Wang, Q.; Tsuji, I. Effects of Maternal Multimicronutrient Supplementation on the Mental Development of Infants in Rural Western China: Follow-up Evaluation of a Double-Blind, Randomized, Controlled Trial. Pediatrics 2009, 123, e685-e692. [CrossRef] [PubMed]

61. McGrath, N.; Bellinger, D.; Robins, J.; Msamanga, G.I.; Tronick, E.; Fawzi, W.W. Effect of Maternal Multivitamin Supplementation on the Mental and Psychomotor Development of Children Who Are Born to HIV-1-Infected Mothers in Tanzania. Pediatrics 2006, 117, e216-e225. [CrossRef] [PubMed]

62. Mulder, K.A.; King, D.J.; Innis, S.M. Omega-3 fatty acid deficiency in infants before birth identified using a randomized trial of maternal DHA supplementation in pregnancy. PLoS ONE 2014, 9, e83764. [CrossRef] [PubMed]

63. Tofail, F.; Kabir, I.; Hamadani, J.; Chowdhury, F.; Yesmin, S.; Mehreen, F.; Huda, S. Supplementation of Fish-oil and Soy-oil during Pregnancy and Psychomotor Development of Infants. J. Health Popul. Nutr. 2006, 24, 48-56. [PubMed]

64. Tofail, F.; Persson, L.; El Arifeen, S.; Hamadani, J.; Mehrin, F.; Ridout, D.; Ekstrom, E.; Huda, S.; Grantham-McGregor, S. Effects of prenatal food and micronutrient supplementation on infant development: A randomized trial from the Maternal and Infant Nutrition Interventions, Matlab (MINIMat) study. Am. J. Clin. Nutr. 2008, 87, 704-711. [PubMed]

65. Zhou, S.; Gibson, R.; Crowther, C.A.; Baghurst, P.; Makrides, M. Effect of iron supplementation during pregnancy on the intelligence quotient and behavior of children at $4 \mathrm{y}$ of age: Long-term follow-up of a randomized controlled trial. Am. J. Clin. Nutr. 2006, 83, 1112-1117. [PubMed]

66. Catena, A.; Muñoz-Machicao, J.A.; Torres-Espínola, F.J.; Martínez-Zaldívar, C.; Diaz-Piedra, C.; Gil, A.; Haile, G.; Gyorei, E.; Molloy, A.M.; Decsi, T.; et al. Folate and long-chain polyunsaturated fatty acid supplementation during pregnancy has long-term effects on the attention system of 8.5-y-old offspring: A randomized controlled trial. Am. J. Clin. Nutr. 2016, 103, 115-127. [CrossRef] [PubMed]

67. Christian, P.; Kim, J.; Mehra, S.; Shaikh, S.; Ali, H.; Shamim, A.A.; Wu, L.; Klemm, R.; Labrique, A.B.; West, K.P. Effects of prenatal multiple micronutrient supplementation on growth and cognition through $2 \mathrm{y}$ of age in rural Bangladesh: The JiVitA-3 Trial. Am. J. Clin. Nutr. 2016, 104, 1175-1182. [CrossRef] [PubMed]

68. Hurtado, J.A.; Iznaola, C.; Pena, M.; Ruiz, J.; Pena-Quintana, L.; Kajarabille, N.; Rodriguez-Santana, Y.; Sanjurjo, P.; Aldamiz-Echevarria, L.; Ochoa, J.; et al. Effects of Maternal Omega-3 Supplementation on Fatty Acids and on Visual and Cognitive Development. J. Pediatr. Gastroenterol. Nutr. 2015, 61, 472-480. [CrossRef] [PubMed]

69. Schmidt, M.K.; Muslimatun, S.; West, C.E.; Schultink, W.; Hautvast, J.G. Mental and psychomotor development in Indonesian infants of mothers supplemented with vitamin A in addition to iron during pregnancy. Br. J. Nutr. 2004, 91, 279-286. [CrossRef] [PubMed]

70. Zhou, S.J.; Skeaff, S.A.; Ryan, P.; Doyle, L.W.; Anderson, P.J.; Kornman, L.; McPhee, A.J.; Yelland, L.N.; Makrides, M. The effect of iodine supplementation in pregnancy on early childhood neurodevelopment and clinical outcomes: Results of an aborted randomised placebo-controlled trial. Trials 2015, 16, 563. [CrossRef] [PubMed]

71. Christian, P.; Murray-Kolb, L.E.; Khatry, S.K.; Katz, J.; Schaefer, B.A.; Cole, P.M.; Leclerq, S.C.; Tielsch, J.M. Prenatal micronutrient supplementation and intellectual and motor function in early school-aged children in Nepal. JAMA 2010, 304, 2716-2723. [CrossRef] [PubMed]

72. Brei, C.; Stecher, L.; Brunner, S.; Ensenauer, R.; Heinen, F.; Wagner, P.; Hermsdorfer, J.; Hauner, H. Impact of the $n-6: n-3$ long-chain PUFA ratio during pregnancy and lactation on offspring neurodevelopment: 5-year follow-up of a randomized controlled trial. Eur. J. Clin. Nutr. 2017, 71, 1114-1120. [CrossRef] [PubMed]

73. Chang, S.; Zeng, L.; Brouwer, I.D.; Kok, F.J.; Yan, H. Effect of iron deficiency anemia in pregnancy on child mental development in rural China. Pediatrics 2013, 131, e755-e763. [CrossRef] [PubMed] 
74. Ramakrishnan, U.; Gonzalez-Casanova, I.; Schnaas, L.; DiGirolamo, A.; Quezada, A.D.; Pallo, B.C.; Hao, W.; Neufeld, L.M.; Rivera, J.A.; Stein, A.D.; et al. Prenatal supplementation with DHA improves attention at 5 y of age: A randomized controlled trial. Am. J. Clin. Nutr. 2016, 104, 1075-1082. [CrossRef] [PubMed]

75. Srinivasan, K.; Thomas, T.; Kapanee, A.R.M.; Ramthal, A.; Bellinger, D.C.; Bosch, R.J.; Kurpad, A.V.; Duggan, C. Effects of maternal vitamin B12 supplementation on early infant neurocognitive outcomes: A randomized controlled clinical trial. Mater. Child Nutr. 2017, 13. [CrossRef] [PubMed]

76. Vuori, L.; Christiansen, N.; Clement, J.; Mora, J.; Wagner, M.; Herrera, M. Nutritional supplementation and the outcome of pregnancy. II. Visual habituation at 15 days. Am. J. Clin. Nutr. 1979, 32, 463-469. [PubMed]

77. World Health Organisation. Case Definition for Acquired Immune Deficiency Syndrome (AIDS); World Health Organisation: Geneva, Switzerland, 1986; pp. 69-73.

78. Pelphrey, K.; Reznick, J.; Goldman, B.D.; Sasson, N.; Morrow, J.; Donahoe, A.; Hodgson, K. Development of visuospatial short-term memory in the second half of the first year. Dev. Psychol. 2004, 40, 836-851. [CrossRef] [PubMed]

79. Diamond, A.; Doar, B. The performance of human infants on a measure of frontal cortex function, delayed response task. Dev. Psychol. 1989, 22, 271-294. [CrossRef] [PubMed]

80. Schwartz, B.; Reznick, J. Measuring infant spatial working memory using a modified delayed-response procedure. Memory 1999, 7, 1-17. [CrossRef] [PubMed]

81. Meltzoff, A.; Moore, M. Newborn infants imitate adult facial gestures. Child Dev. 1983, 54, 702-709. [CrossRef] [PubMed]

82. Fenson, L.; Marchman, V.; Thal, D.; Dale, P.; Reznick, J.; Bates, E. The MacArthur-Bates Communicative Development Inventories: User's Guide and Technical Manual, 2nd ed.; Paul H. Brookes Publishing Co.: Baltimore, MD, USA, 2007.

83. Mullen, E. Mullen Scales of Early Learning, AGS Edition: Manual and Item Assessment Book; American Guidance Service Inc.: Circle Pines, MN, USA, 1995.

84. Thorndike, R.; Hagen, E.; Sattler, J. Stanford-Binet Intelligence Scale: Technical Manual, 4th ed.; The Riverside Publishing Company: Itasca, IL, USA, 1986.

85. Goodman, R. Strengths and Difficulties Questionnaire 1999. Available online: http://www.sdqinfo.com/ (accessed on 25 January 2015).

86. Bayley, N. Bayley Scales of Infant Development, 2nd ed.; Psychological Corporation: San Antonio, TX, USA, 1993.

87. Huang, H.; Tao, S.; Zhang, Y. Standarization of Bayley scales of infant development in Shanghai. Chin. J. Child Health 1993, 1, 158-160.

88. Ireton, H. Child Development Inventory Manual; Behavior Science Systems: Minneapolis, MN, USA, 1992.

89. Brandstetter, G.; Siebler, V.; Schneider, H.; Grässle, A.; Steinmacher, J.; Bode, H. Elternfragebogenzur Entwicklung im Kleinkindalter (EFkE) -Ein Screeninginstrument: I. Normierung Kinderarztl Prax 2002, 5, 338-344.

90. Hermsdorfer, J.; Mai, N.; Marquardt, C. Evaluation of precision grip using pneumatically controlled loads. J. Neurosci. Methods 1992, 45, 117-126. [CrossRef]

91. Uttner, I.; Mai, N.; Esslinger, O.; Danek, A. Quantitative evaluation of mirror movements in adults with focal brain lesions. Eur. J. Neurol. 2005, 12, 964-975. [CrossRef] [PubMed]

92. Griffiths, R. The abilities of young children. In A Comprehensive System of Mental Measurement for the First Eight Years of Life; Child Development Research Centre: London, UK, 1970.

93. Dunn, L.M.; Dunn, L. Peabody Picture Vocabulary Test-Revised (PPVT-R); American Guidance Service: Pine Circle, MN, USA, 1981.

94. Achenbach, T. Manual for the Child Behaviour Checklist 1.5-5 and 1991 Profile; University of Vermont Department of Psychiatry: Burlington, VT, USA, 1991.

95. Colombo, J.; Kannass, K.; Shaddy, D.; Kundurthi, S.; Maikranz, J.; Anderson, C.J.; Blaga, O.M.; Carlson, S.E. Maternal DHA and the development of attention in infancy and toddlerhood. Child Dev. 2004, 75, 1254-1267. [CrossRef] [PubMed]

96. Kannass, K.; Colombo, J.; Carlson, S. Maternal DHA levels and toddler free-play attention. Dev. Neuropsychol. 2009, 34, 159-174. [CrossRef] [PubMed]

97. Sparrow, S.; Balla, D.; Cicchetti, D. Vineland Adaptive Behavior Scales: Interview Edition, Survey Form Manual; American Guidance Service: Circle Pines, MN, USA, 1984. 
98. Fagan, J.; Detterman, D. The Fagan test of infant intelligence: A technical summary. J. Appl. Dev. Psychol. 1992, 13, 173-193. [CrossRef]

99. Kaufman, A.S.; Kaufman, N.L. Kaufman Assessment Battery for Children, 2nd ed.; American Guidance Service: Circle Pines, MN, USA, 2004.

100. Willatts, P. Beyond the couch potato infant: How infants use their knowledge to regulate action, solve problems and achieve goals. In Infant Development: Recent Advances Hove; Bremmer, J.G., Slater, A., Butterworth, G., Eds.; Psychology Press: Hove, UK, 1997; pp. 109-135.

101. Willatts, P. Development of means-end behavior in young infants: Pulling a support to retrieve a distant object. Dev. Psychol. 1999, 35, 651-667. [CrossRef] [PubMed]

102. Willatts, P. The stage-IV infant's solution of problems requiring the use of supports. Infant Behav. Dev. 1984, 7, 125-134. [CrossRef]

103. Willatts, P. Stages in the development of intentional search by young infants. Dev. Psychol. 1984, 20, 389-396. [CrossRef]

104. McCarthy, D. Manual for the McCarthy scales of children's abilities. In The Psychological Corporation; Harcourt Brace Jovanovich Inc.: New York, NY, USA, 1972.

105. Reynolds, C.R.; Kamphaus, R.W. The Clinician's Guide to the Behavior Assessment System for Children (BASC); The Guilford Press: New York, NY, USA, 2002.

106. Conners, C.K. Conners' Continuous Performance Test II: Computer Program for Windows Technical Guide and Software Manual; Multi-Health Systems Inc.: Tonawanda, NY, USA, 2000.

107. Wolfe, D.; Skuse, D.; Mathisen, V. Behavioral style in failure to thrive infants: A preliminary communication. J. Pediatr. Psychol. 1990, 15, 237-254.

108. Rueda, M.R.; Fan, J.; McCandliss, B.D.; Halparin, J.D.; Gruber, D.B.; Lercari, L.P.; Posner, M.I. Development of attentional networks in childhood. Neuropsychologia 2004, 42, 1029-1040. [CrossRef] [PubMed]

109. Fan, J.; McCandliss, B.D.; Sommer, T.; Raz, A.; Posner, M.I. Testing the efficiency and independence of attentional networks. J. Cogn. Neurosci. 2002, 14, 340-347. [CrossRef] [PubMed]

110. Bracken, B.A.; McCallum, R.S. Universal Non-Verbal Intelligence Test (UNIT); PRO-ED: Austin, TX, USA, 1998.

111. Konishi, S.; Nakajima, K.; Uchida, I.; Sekihara, K.; Miyashita, Y. No-go dominant brain activity in human inferior prefrontal cortex revealed by functional magnetic resonance imaging. Eur. J. Neurosci. 1998, 10, 1209-1213. [CrossRef] [PubMed]

112. Bull, R.; Scerif, G. Executive functioning as a predictor of children's mathematics ability: Inhibition, switching, and working memory. Dev. Neuropsychol. 2001, 19, 273-293. [CrossRef] [PubMed]

113. Wechsler, D. Wechsler Preschool and Primary Scale of Intelligence, 3rd ed.; Harvcourt Assessment Inc.: San Antonio, TX, USA, 2002.

114. Henderson, S.E.; Sugden, D.A. Movement Assessment Battery for Children (MABC); Psychological Corp: London, UK, 1992.

115. Reitan, R.M.; Wolfson, D. The Halstead-Reitan Neuropsychological Test Battery: Theory E Clinical Interpretation; Neuropsychology Press: Tucson, AZ, USA, 1985.

116. Joos, S.K.; Pollitt, E.; Mueller, W.H.; Albright, D.L. The Bacon Chow Study: Maternal Nutritional Supplementation and Infant Behavioral Development. Child Dev. 1983, 54, 669-676. [CrossRef] [PubMed]

117. Bayley, N. Manual for Bayley Scales of Infant Development, 1st ed.; Psychological Corp: New York, NY, USA, 1969.

118. Squires, J.; Potter, L.; Bricker, D. The Ages and Stages Questionnaire User's Guide, 2nd ed.; Paul H. Brookes Publishing, Co.: Baltimore, MD, USA, 1999.

119. Dunn, L.M.; Dunn, L.; Whetton, C.; Burley, J. British Picture Vocabulary Scale, 2nd ed.; NFER-Nelson Publishing Co., Ltd.: London, UK, 1997.

120. Elliot, C. British Ability Scales, 2nd ed.; NFER-Nelson Publishing Co., Ltd.: London, UK, 1996.

121. Korkman, M.; Kirk, U.; Kemp, S. NEPSY: A Developmental Neuropsychological Assessment; The Psychological Corporation: Orlando, FL, USA, 1998.

122. Kochanska, G.; Murray, K.; Harlan, E. Eftortful control in early childhood: Continuity and change, antecedents and implications for social development. Dev. Psychol. 2000, 36, 220-232. [CrossRef] [PubMed]

123. Carlson, S.M. Developmentally sensitive measures of executive function in preschool children. Dev. Neuropsychol. 2005, 28, 595-616. [CrossRef] [PubMed]

124. Russell, J.; Mauthner, N.; Sharpe, S.; Tidswell, T. The 'windows task' as a measure of strategic deception in preschoolers and autistic subjects. Br. J. Dev. Psychol. 1991, 9, 331-349. [CrossRef] 
125. Waber, D.P.; Vuori Christiansen, L.; Ortiz, N.; Clement, J.R.; Christiansen, N.E.; Mora, J.O.; Reed, R.B.; Herrera, M.G. Nutritional supplementation, maternal education, and cognitive development of infants at risk of malnutrition. Am. J. Clin. Nutr. 1981, 34, 807-813. [PubMed]

126. Corman, H.; Escalona, S. Stages of sensorimotor development: A replication study. Merrill-Palmer Q. 1969, 15, 351-361.

127. Elliott, C.D. Differential Ability Scales Mannual 2: Technical Handbook; National Foundation for Educational Research-Nelson: Berkshire, UK, 1983.

128. Kirk, S.; McCarthy, J.; Kirk, W. Illinois Test of Pycholinguistic Abilities (Revised); University of Illinois Press: Champaign, IL, USA, 1968.

129. Stone, M.; Wright, B. Knox's Cube Test; Stoelting Company: Chicago, IL, USA, 1980.

130. Folio, M.; Fewell, R. Peabody Development Motor Scales and Acuity Cards; DLM Teaching Resources: Allen, TX, USA, 1983.

131. Wilson, B.; Lacoviello, J.; Wilson, J.; Risucci, D. Purde pegboard performance of normal children. J. Clin. Neurophysiol. 1982, 4, 19-26.

132. Fein, G.G. Toys and stories. In The Future of Play Theory: A Multidisciplinary Inquiry into the Contributions of Brian Sutton-Smith; Pellegrini, A.D., Ed.; State University of New York Press: Albany, NY, USA, 1995.

133. Morrow, L. Effects of structural guidance in story telling on children's dictation of original stories. J. Read. Behav. 1986, 18, 135-152. [CrossRef]

134. Harris, D. Children's Drawings as Measures of Intellectual Maturity: A Revision and Extension of the Goodenough Draw-a-Man Test; Harcourt, Brace and World: New York, NY, USA, 1963.

135. Selman, R. The Growth of Interpersonal Understanding: Developmental and Clinical Analyses; Academic Press: New York, NY, USA, 1980.

136. Serafica, F.C. Conceptions of friendship and interaction between friends: An Organismic-Developmental Perspective. In Social Cognitive Development in Context; Serafica, F.C., Ed.; The Guilford Press: New York, NY, USA, 1982.

137. Behar, L.; Stringfield, S. A behaviour rating scale for the preschool child. Dev. Psychol. 1974, 10, 601-610. [CrossRef]

138. Isaacs, E.; Oates, J. Nutrition and cognition assessing cognitive abilities in children and young people. Eur. J. Nutr. 2008, 47, 4-24. [CrossRef] [PubMed]

139. Wainwright, P.E.; Colombo, J. Nutrition and the development of cognitive functions: Interpretation of behavioral studies in animals and human infants. Am. J. Clin. Nutr. 2006, 84, 961-970. [PubMed]

140. Yetley, E.A. Multivitamin and multimineral dietary supplements: Definitions, characterization, bioavailability, and drug interactions. Am. J. Clin. Nutr. 2007, 85, 269S-276S. [PubMed]

141. Huang, H.Y.; Appel, L.J. Supplementation of Diets with $\alpha$-Tocopherol Reduces Serum Concentrations of $\gamma$ and $\delta$-Tocopherol in Humans. J. Nutr. 2003, 133, 3137-3140. [PubMed]

142. Cheatham, C.; Sheppard, K. Synergistic Effects of Human Milk Nutrients in the Support of Infant Recognition Memory: An Observational Study. Nutrients 2015, 7, 9079-9095. [CrossRef] [PubMed]

143. Black, M.M. Effects of Vitamin B12 and Folate Deficiency on Brain Development in Children. Food Nutr. Bull. 2008, 29, S126-S131. [CrossRef] [PubMed]

144. Benton, D. The influence of dietary status on the cognitive performance of children. Mol. Nutr. Food Res. 2010, 54, 457-470. [CrossRef] [PubMed]

145. Nyaradi, A.; Jiang Hong, L.; Hickling, S.; Foster, J.; Oddy, W.H. The role of nutrition in children's neurocognitive development, from pregnancy through childhood. Front. Hum. Neurosci. 2013, 7, 1-16. [CrossRef] [PubMed]

146. Muller, O.; Krawinkel, M. Malnutrition and health in developing countries. CMAJ 2005, 173, $279-286$. [CrossRef] [PubMed]

(C) 2017 by the authors. Licensee MDPI, Basel, Switzerland. This article is an open access article distributed under the terms and conditions of the Creative Commons Attribution (CC BY) license (http:/ / creativecommons.org/licenses/by/4.0/). 